\title{
NUMERICAL VALUES OF SOME INTEGRALS INVOLVING BESSEL FUNCTIONS
}

\author{
by D. L. GEORGE $\dagger$ \\ (Received 1st July, 1961)
}

\section{Introduction}

In the solution of boundary value problems in mathematical physics by means of integral transforms, we often find that the solution of a particular problem can be expressed in terms of integrals of the type

$$
\begin{aligned}
& S(v, n)=\int_{0}^{\infty} u^{n} \sin u J_{v}(u r) e^{-z u} d u, \ldots \ldots \ldots . . . \\
& C(v, n)=\int_{0}^{\infty} u^{n-1}(1-\cos u) J_{v}(u r) e^{-z u} d u,
\end{aligned}
$$

where $r$ and $z$ are positive, and $v$ and $n$ are integers satisfying the convergence condition $v+n>-1$.

The method of evaluating integrals of this type is outlined by Sneddon (1, pp. 466-468) but he does not give tables of numerical values though in the paper (2) he gives tables of stress components depending directly on these integrals.

The object of the present paper is to make available tables of numerical values of the integrals $S(v, n), C(v, n)$ for the values:

$$
\begin{array}{ll}
v=0, & n=0,1 ; \\
v=1, & n=-1,0,1 .
\end{array}
$$

Analytical expressions for these integrals are derived in $\$ \S 2,3$ below. These integrals may be regarded as basic, not only in the sense that they are the ones which occur most frequently in applications but also because values of integrals of these types with higher values of $v$ or $n$ can be derived by means of the recurrence relations of $\S 4$.

Tables of values of the ten basic integrals listed above for values of $r, z$ from 0 to 2 by steps of $0 \cdot 1$ and from 2 to 10 by unit steps are given in tables 1-10. The numerical calculations were carried out using the IBM 650 Digital Computer at Duke University.

$\dagger$ The work described here was done at Duke University, North Carolina and was supported by the U.S. Air Force Office of Scientific Research, A.R.D.C., under Contract AF 18(600)-1341. 


\section{Expressions for the Integrals $S(v, n)$}

The method of evaluating the integrals is illustrated by Pétiau $(3$, p. 51$)$ who deduces the value of $S(0,0)$ by putting $p=z-i$ in the well-known formula

$$
\int_{0}^{\infty} J_{0}(r u) e^{-p u} d u=\left(r^{2}+p^{2}\right)^{-\frac{1}{2}}
$$

and equating imaginary parts. In this way we find that

where we have written

$$
S(0,0)=\frac{Y}{X^{2}+Y^{2}}
$$

$$
\begin{aligned}
& 2 X^{2}=\left[\left(r^{2}+z^{2}-1\right)^{2}+4 z^{2}\right]^{\frac{1}{2}}+\left(r^{2}+z^{2}-1\right), \\
& 2 Y^{2}=\left[\left(r^{2}+z^{2}-1\right)^{2}+4 z^{2}\right]^{\frac{1}{2}}-\left(r^{2}+z^{2}-1\right) .
\end{aligned}
$$

In a similar way from the formulæ

$$
\begin{aligned}
& \int_{0}^{\infty} u J_{0}(r u) e^{-p u} d u=p\left(r^{2}+p^{2}\right)^{-\frac{3}{2}}, \ldots \ldots \ldots . \\
& \int_{0}^{\infty} u^{-1} J_{1}(r u) e^{-p u} d u=r^{-1}\left[\left(r^{2}+p^{2}\right)^{\frac{1}{2}}-p\right], \\
& \int_{0}^{\infty} J_{1}(r u) e^{-p u} d u=r^{-1}\left[1-p\left(r^{2}+p^{2}\right)^{-\frac{1}{2}}\right], \ldots \\
& \int_{0}^{\infty} u J_{1}(r u) e^{-p u} d u=r\left(r^{2}+p^{2}\right)^{-\frac{2}{2}}, \ldots \ldots \ldots \ldots
\end{aligned}
$$

$(4$, p. 112) we deduce that

$$
\begin{aligned}
& S(0,1)=\frac{3 X Y(z X+Y)-\left(z Y^{3}+X^{3}\right)}{\left(X^{2}+Y^{2}\right)^{3}}, \\
& S(1,-1)=\frac{1-Y}{r}, \\
& S(1,0)=\frac{X-z Y}{r\left(X^{2}+Y^{2}\right)}, \\
& S(1,1)=\frac{r Y\left(3 X^{2}-Y^{2}\right)}{\left(X^{2}+Y^{2}\right)^{3}}
\end{aligned}
$$

When $z=0, S(0,1)$ and $S(1,1)$ are divergent and

$$
\begin{array}{r}
S(0,0)=\left\{\begin{array}{lr}
\left(1-r^{2}\right)^{-\frac{1}{2}}, & 0<r<1 \\
0, & r>1
\end{array}\right. \\
S(1,-1)=\left\{\begin{array}{lr}
r^{-1}\left[1-\left(1-r^{2}\right)^{\frac{1}{2}}\right], & 0<r<1 \\
r^{-1}, & r>1
\end{array}\right.
\end{array}
$$




$$
S(1,0)=\left\{\begin{array}{lr}
0 & 0<r<1 \\
r^{-1}\left(r^{2}-1\right)^{-\frac{1}{2}} & r>1
\end{array}\right.
$$

When $r=0, S(1,-1), S(1,0)$ and $S(1,1)$ are all zero and

$$
\begin{aligned}
& S(0,0)=\left(1+z^{2}\right)^{-1}, \\
& S(0,1)=2 z\left(1+z^{2}\right)^{-2} .
\end{aligned}
$$

It should also be noted that

$$
\lim _{r \rightarrow 0} r^{-1} S(1, n)=\frac{1}{2}[S(0, n+1)]_{r}=0 .
$$

\section{Expressions for the Integrals $C(v, n)$}

We can derive expressions by which to calculate the integrals $C(v, n)$ by an exactly similar method.

From equations (3), (8) and (9) we find immediately that

$$
\begin{aligned}
& C(0,1)=\left(r^{2}+z^{2}\right)^{-\frac{1}{2}}-\frac{X}{X^{2}+Y^{2}}, \\
& C(1,0)=r^{-1}\left[\left(r^{2}+z^{2}\right)^{\frac{1}{2}}-X\right], \quad \ldots \\
& C(1,1)=\frac{z X+Y}{X^{2}+Y^{2}}-\frac{z}{r \sqrt{ }\left(r^{2}+z^{2}\right)}
\end{aligned}
$$

Further, from equation (3) we find by integrating both sides with respect to $p$ from $z$ to $p$ that

$$
\int_{0}^{\infty} u^{-1}\left[e^{-z u}-e^{-p u}\right] J_{0}(u r) d u=\log \left[p+\left(r^{2}+p^{2}\right)^{\frac{1}{2}}\right]-\log \left[z+\left(r^{2}+z^{2}\right)^{\frac{1}{1}}\right] .
$$

Making the substitution $p=z-i$ and equating real parts we obtain the expression

$$
\left.C(0,0)=\frac{1}{2} \log \left[(z+X)^{2}+(1+Y)^{2}\right]-\log \left[z+r^{2}+z^{2}\right)^{\frac{1}{2}}\right]
$$

Similarly integrating both sides of equation (8) with respect to $p$ from $z$ to $z-i$ and equating real parts we obtain the expression

$$
\begin{aligned}
C(1,-1)= & \frac{1}{2 r}\left\{1-Y+z\left[X-\left(r^{2}+z^{2}\right)^{\frac{1}{1}}\right]\right\} \\
& +\frac{1}{4} r \log \left[(z+X)^{2}+(1+Y)^{2}\right]-\frac{1}{2} r \log \left[z+\left(r^{2}+z^{2}\right)^{\frac{1}{1}}\right] .
\end{aligned}
$$

When $z=0$ we have the formula

$$
\begin{aligned}
C(0,0) & =\left\{\begin{array}{lr}
\log \left[1+\left(1-r^{2}\right)^{\frac{1}{2}}\right]-\log r, & 0<r<1 \\
0, & r>1
\end{array}\right. \\
C(0,1) & =\left\{\begin{array}{lr}
r^{-1}, & 0<r<1 \\
r^{-1}-\left(r^{2}-1\right)^{-\frac{1}{2}}, & r>1
\end{array}\right.
\end{aligned}
$$




$$
\begin{aligned}
C(1,-1) & =\left\{\begin{array}{lr}
\frac{1}{2 r}\left[1-\left(1-r^{2}\right)^{\frac{1}{2}}\right]+\frac{1}{2} r\left\{\log \left[1+\left(1-r^{2}\right)^{\frac{1}{2}}\right]-\log r\right\}, & 0<r<1 \\
\frac{1}{2 r}, & r>1
\end{array}\right. \\
C(1,0) & =\left\{\begin{array}{lr}
1, & 0<r<1 \\
1-r^{-1}\left(r^{2}-1\right)^{\frac{1}{2}}, & r>1
\end{array}\right. \\
C(1,1) & =\left\{\begin{array}{lr}
r^{-1}\left(1-r^{2}\right)^{-\frac{1}{2}} & 0<r<1 \\
0 & r>1
\end{array}\right.
\end{aligned}
$$

for the evaluation of the integrals.

When $r=0(z>0)$ the integrals $C(1,-1), C(1,0)$ and $C(1,1)$ are all zero but it should be noted that

$$
\lim _{r \rightarrow 0} r^{-1} C(1, n)=\frac{1}{2}[C(0, n+1)]_{r=0}
$$

and that for $r=0$,

$$
\begin{aligned}
& C(0,0)=\frac{1}{2} \log \left(1+z^{2}\right)-\log z \\
& C(0,1)=z^{-1}\left(1+z^{2}\right)^{-1}
\end{aligned}
$$

\section{Recurrence Formula}

In some problems we may wish to evaluate integrals of the type $C(v, n)$, $S(v, n)$ where $v, n$ are integers, $v \geqq 2, n \geqq 2$. In this section we derive some simple recurrence relations by means of which such integrals may be found.

Integrals involving Bessel functions of higher order are easily found from the recurrence relation

$$
J_{v+1}(u r)+J_{v-1}(u r)=\frac{2 v}{u r} J_{v}(u r)
$$

(4, p. 95). Inserting this in the definitions (1), (2) we obtain the simple recurrence formulæ

$$
\begin{aligned}
& S(v+1, n)=\frac{2 v}{r} S(v, n-1)-S(v-1, n) \\
& C(v+1, n)=\frac{2 v}{r} C(v, n-1)-C(v-1, n) .
\end{aligned}
$$

Results of this kind for $v=0,1, n \geqq 2$, can be found in a similar way. Since

$$
J_{1}(u r)=-\frac{1}{r} \frac{\partial}{\partial u}\left[J_{0}(u r)\right]
$$

we find, after an integration by parts that

$$
C(1, n+1)=\frac{1}{r} \int_{0}^{\infty} J_{0}(u r) \frac{\partial}{\partial u}\left[u^{n}(1-\cos u) e^{-z u}\right] d u
$$


from which it follows that

$$
C(1, n+1)=\frac{1}{r}\{S(0, n)+n C(0, n)-z C(0, n+1)\} .
$$

On the other hand, using the relation

$$
u J_{0}(u r)=\frac{1}{r} \frac{\partial}{\partial u}\left[u J_{1}(u r)\right]
$$

and integrating by parts, we find that

which is equivalent to

$$
C(0, n+1)=-\frac{1}{r} \int_{0}^{\infty} u J_{1}(u r) \frac{\partial}{\partial u}\left[u^{n-1}(1-\cos u) e^{-z u}\right] d u
$$

$$
C(0, n+1)=\frac{1}{r}\{z C(n, n+1)-(n-1) C(1, n)-S(1, n)\} .
$$

Solving equations (27) and (28) for $C(1, n+1)$ and $C(0, n+1)$ we find that

$$
\begin{aligned}
C(0, n+1) & =\frac{z}{r^{2}+z^{2}}[n C(0, n)+S(0, n)] \\
& =\frac{r}{r^{2}+z^{2}}[(n-1) C(1, n)+S(1, n)] \\
C(1, n+1) & =\frac{z}{r^{2}+z^{2}}[(n-1) C(1, n)+S(1, n)] \\
& +\frac{r}{r^{2}+z^{2}}[n C(0, n)+S(0, n)] . \ldots \ldots \ldots
\end{aligned}
$$

Proceeding just as we did in the derivation of equations (27), (28) we obtain the relations

$$
\begin{aligned}
& r S(1, n+1)+z S(0, \mathrm{n}+1)=(n+1) S(0, n)+I(0, n+1)-C(0, n+2), \\
& z S(1, n+1)-r S(0, n+1)=n S(1, n)+I(1, n+1)-C(1, n+2),
\end{aligned}
$$

where

$$
I(v, n)=\int_{0}^{\infty} u^{n} J_{v}(u r) e^{-u z} d u .
$$

These equations may be solved in turn to give

$$
\begin{aligned}
S(1, n+1) & =\frac{r}{r^{2}+z^{2}}[(n+1) S(0, n)+I(0, n+1)-C(0, n+2)] \\
& +\frac{z}{r^{2}+z^{2}}[n S(1, n)+I(1, n+1)-C(1, n+2)], \ldots \ldots \\
S(0, n+1) & =\frac{z}{r^{2}+z^{2}}[(n+1) S(0, n)+I(0, n+1)-C(0, n+2)] \\
& -\frac{r}{r^{2}+z^{2}}[n S(1, n)+I(1, n+1)-C(1, n+2)\} \ldots \ldots .
\end{aligned}
$$


Since the integrals $C(0, n+2), C(1, n+2)$ can be determined easily from equations (29), (30) and $I(v, n)$ defined by equation (31) can be evaluated by means of the formula

$$
I(v, n)=\frac{\Gamma(n+v+1) r^{v}}{2^{v} \Gamma(v+1)\left(r^{2}+z^{2}\right)^{\frac{1}{2 n+\frac{1}{2} v+\frac{1}{2}}}}{ }_{2} F_{1}\left(\frac{n+v+1}{2}, \frac{v-n}{2} ; v+1 ; \frac{r^{2}}{r^{2}+z^{2}}\right)
$$

$(z>0, v+n>0),(4$, p. 111$)$ we see that $S(0, n+1), S(1, n+1)$ can be found by means of equations (32) and (33).

\section{REFERENCES}

(1) I. N.SNEdDon, Fourier Transforms (McGraw-Hill, New York, 1951).

(2) I. N. Sneddon, Boussinesq's problem for a rigid cone, Proc. Camb. Phil. Soc., 44 (1948), 492.

(3) G. PÉtiau, La Théorie des Fonctions de Bessel (C.N.R.S., Paris, 1955).

(4) I. N. SNEddon, Special Functions of Mathematical Physics and Chemistry. (Interscience, New York, 1956).

Department of Mathematics

North Carolina State College

RALEIGH

North Carolina 
TABLES I-X 


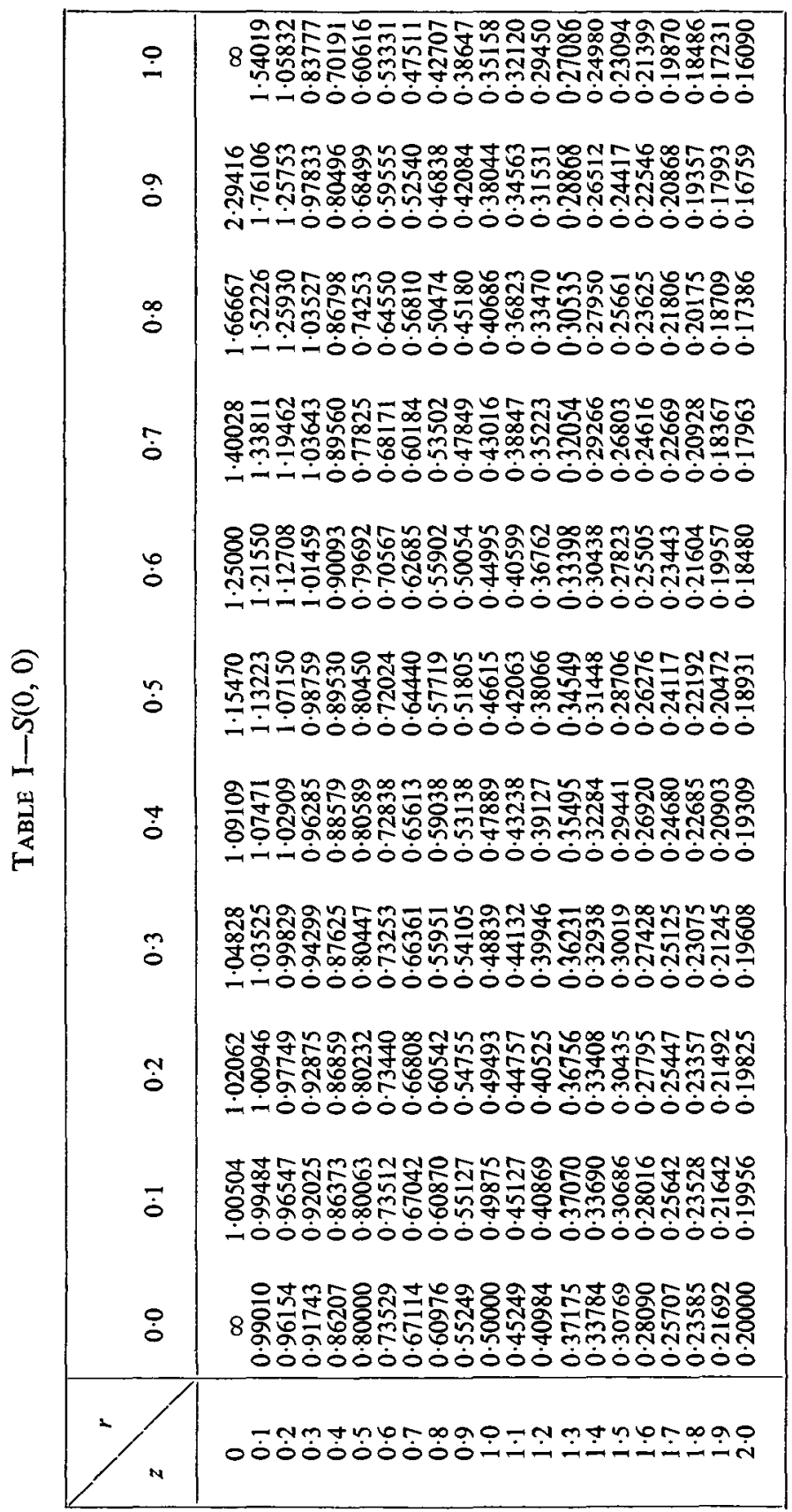




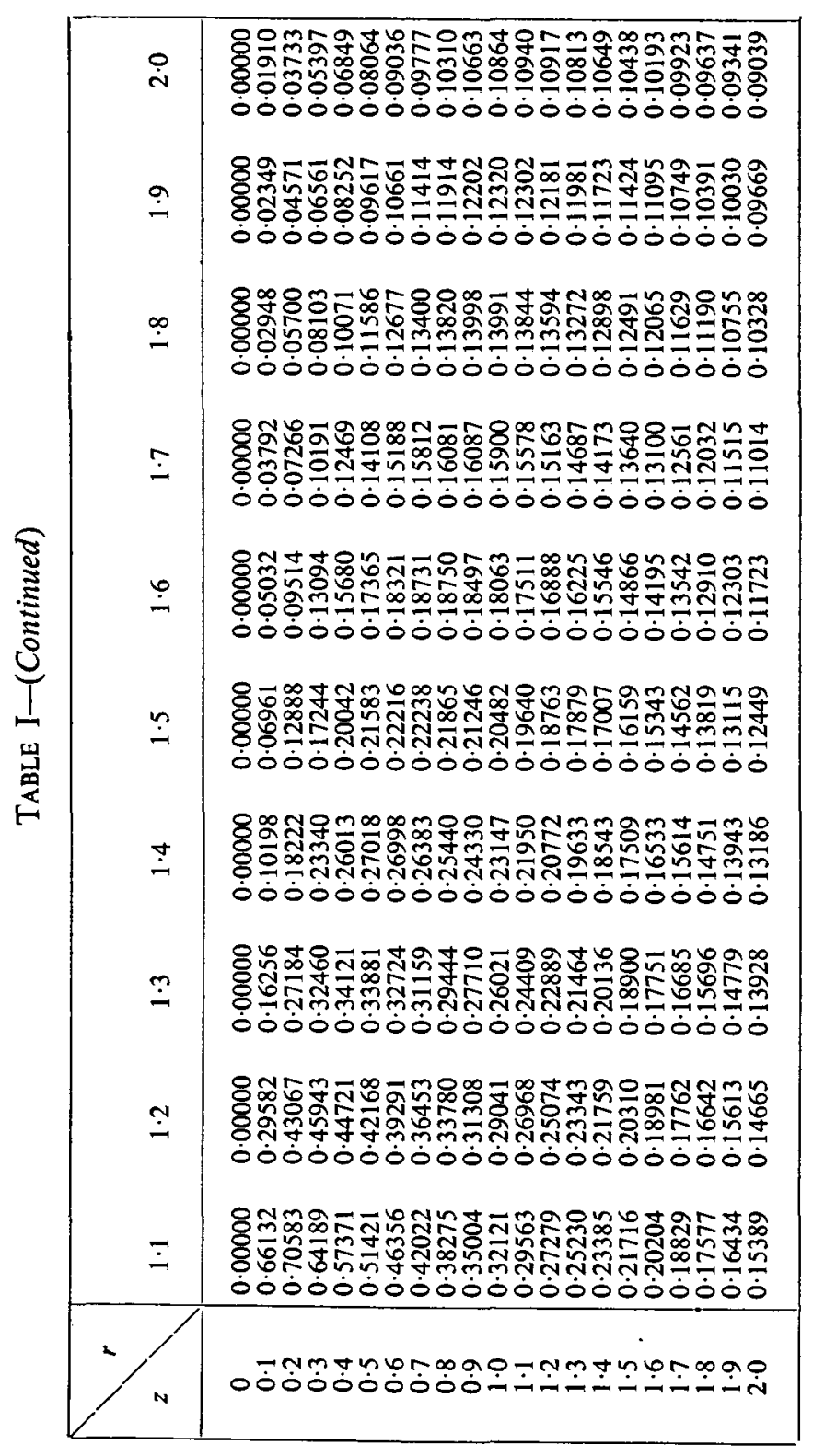

\begin{tabular}{|c|c|}
\hline$\dot{\dot{\theta}}$ & 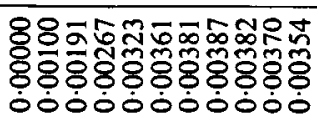 \\
\hline$\stackrel{\circ}{\circ}$ & 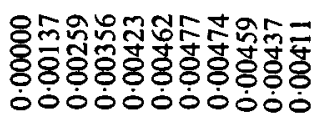 \\
\hline م) & 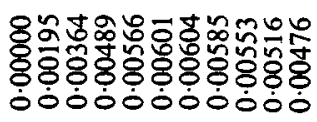 \\
\hline$\stackrel{P}{r}$ & 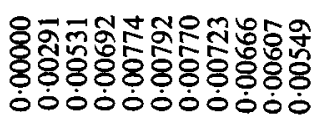 \\
\hline$\dot{0}$ & 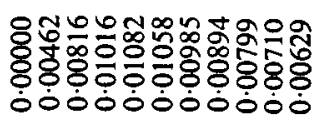 \\
\hline$\dot{\text { in }}$ & 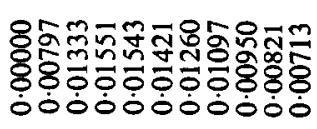 \\
\hline$\dot{\dot{v}}$ & 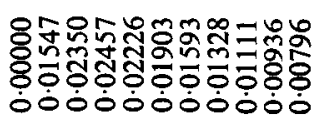 \\
\hline ُْ & 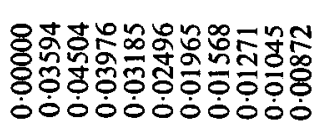 \\
\hline$\stackrel{\text { ஸे }}{\text { N }}$ & 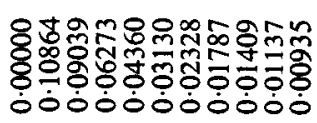 \\
\hline ب) & 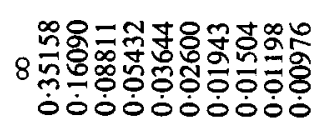 \\
\hline ஜ & 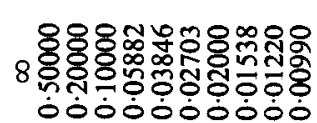 \\
\hline & 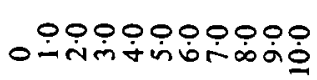 \\
\hline
\end{tabular}




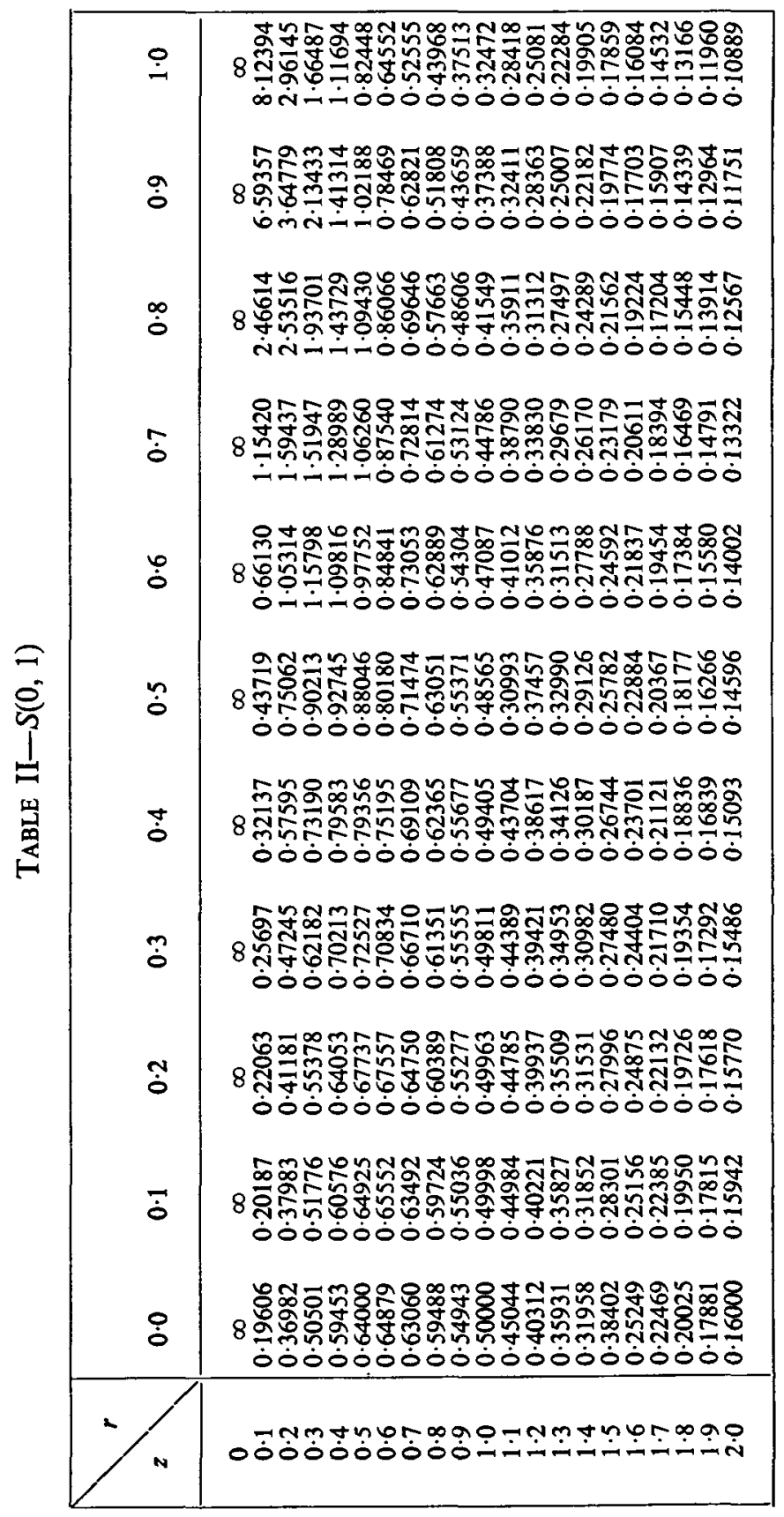




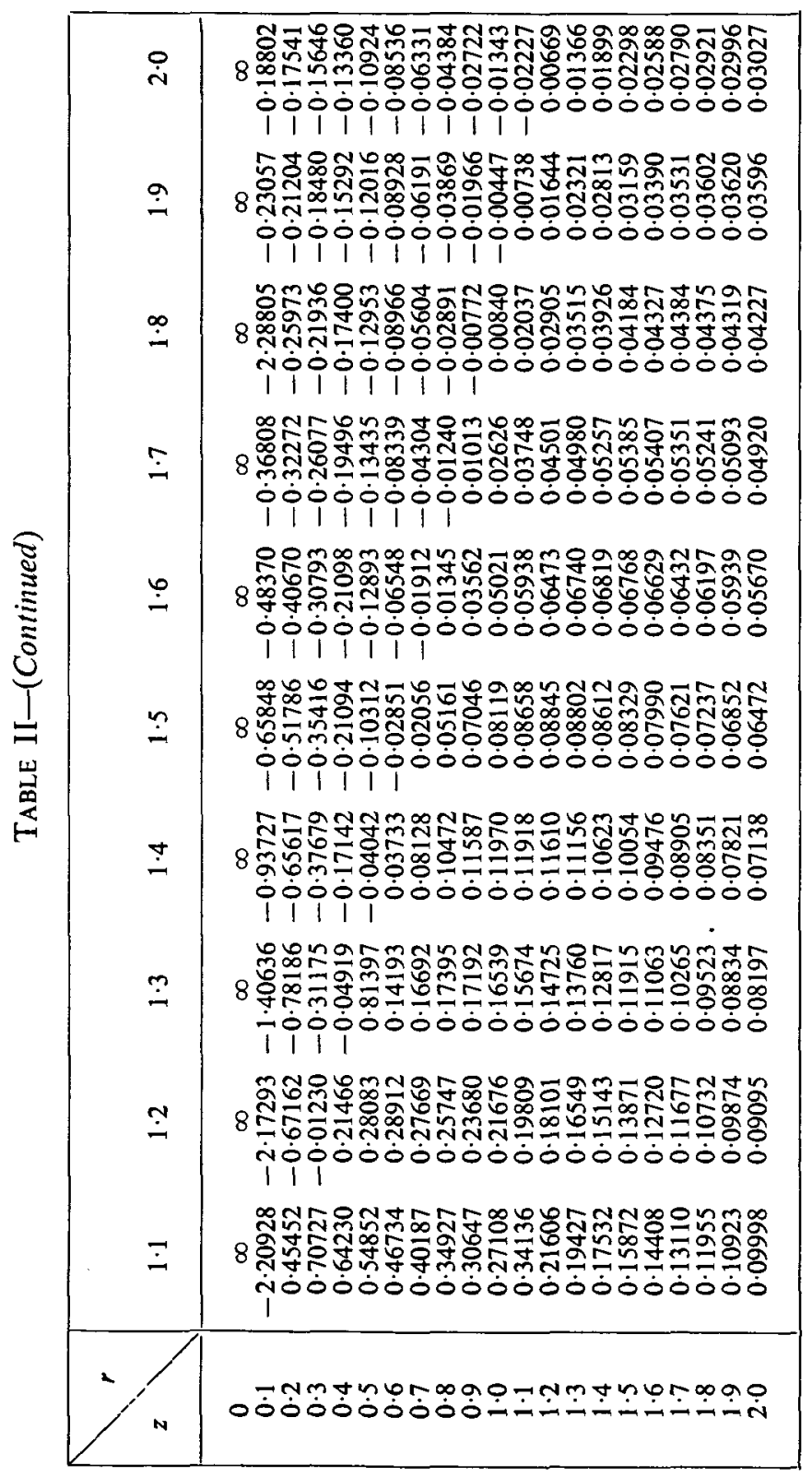

\begin{tabular}{|c|c|}
\hline$\stackrel{\varphi}{\dot{0}}$ & 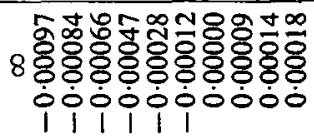 \\
\hline 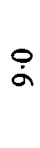 & 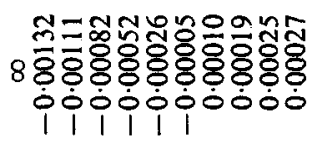 \\
\hline$\stackrel{\varphi}{\dot{\infty}}$ & 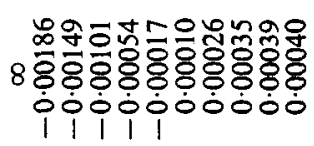 \\
\hline$\stackrel{\varphi}{r}$ & 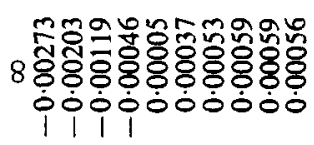 \\
\hline$\dot{\varphi}$ & 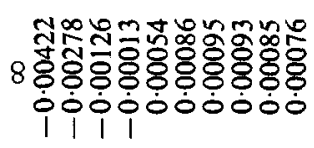 \\
\hline$\stackrel{\varphi}{\dot{v}}$ & 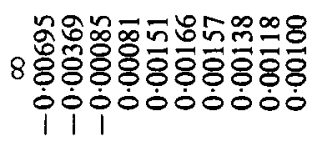 \\
\hline & 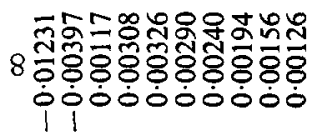 \\
\hline 0 & 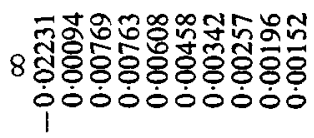 \\
\hline$\stackrel{\leftrightarrow}{\dot{\sim}}$ & 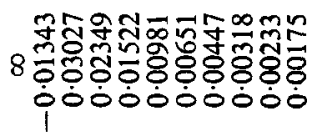 \\
\hline & 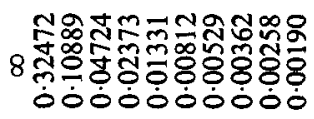 \\
\hline 0 & 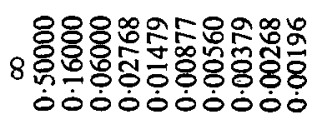 \\
\hline & ○ \\
\hline
\end{tabular}

E.M.S. $-G$ 


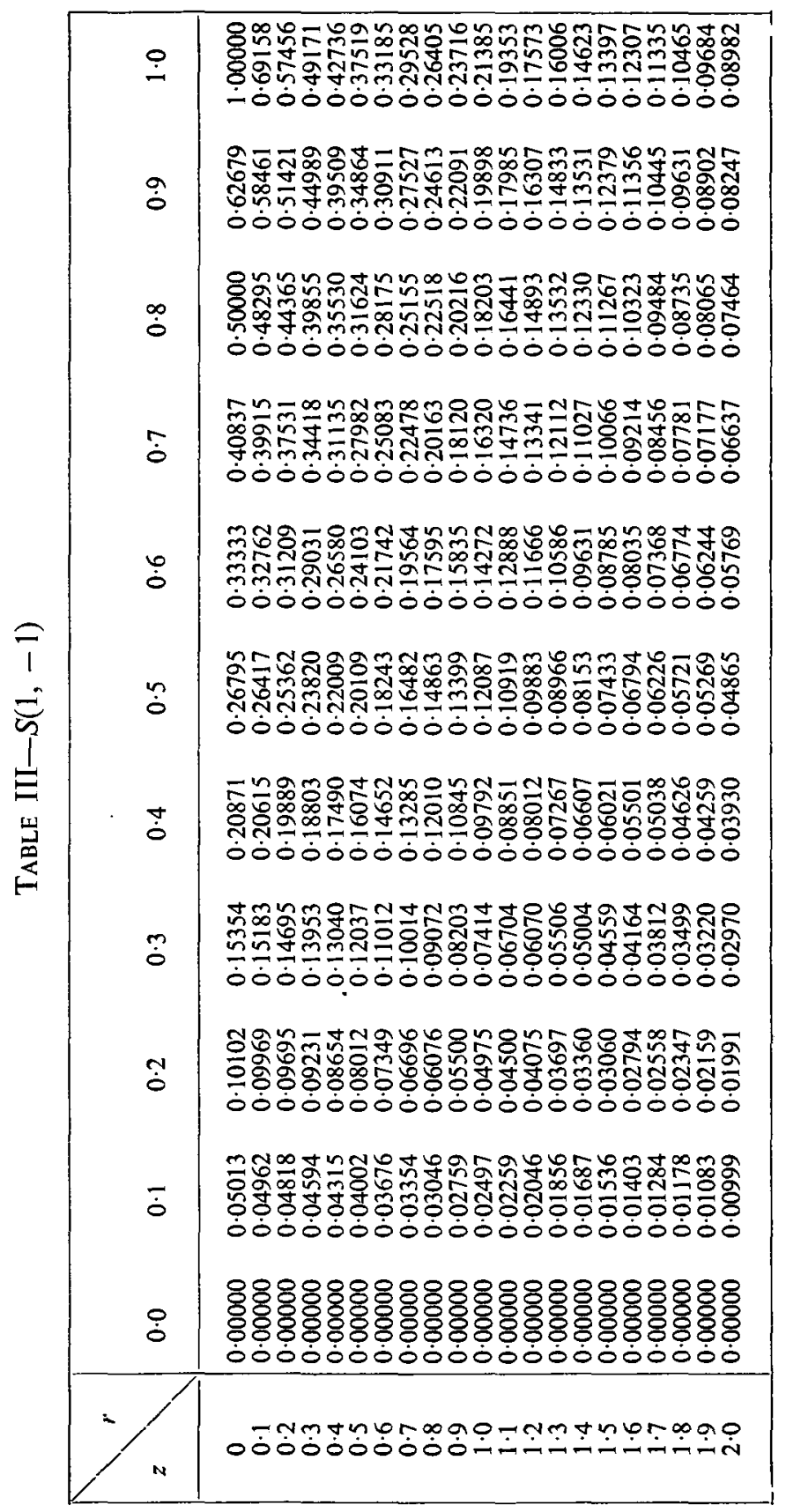




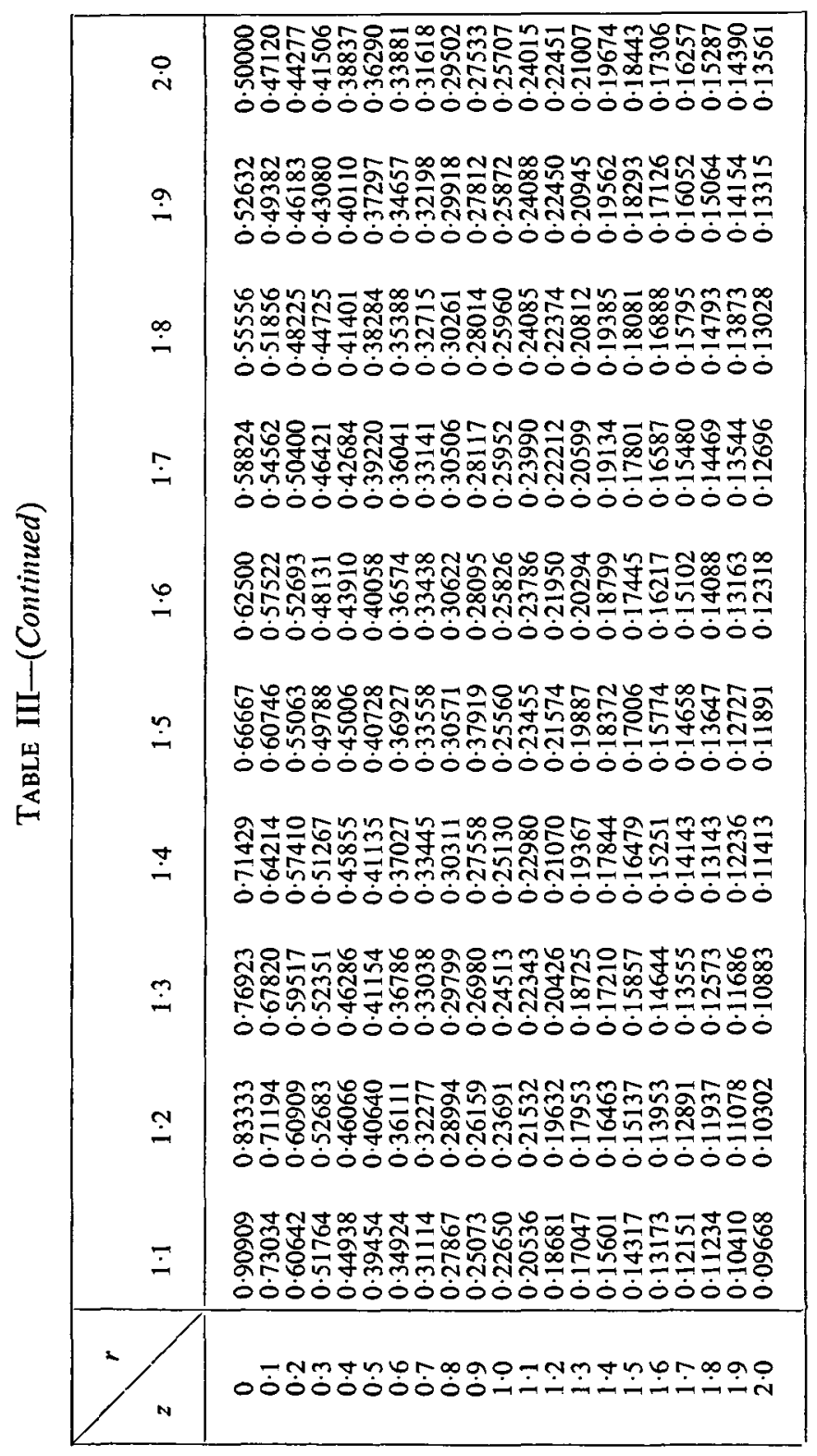

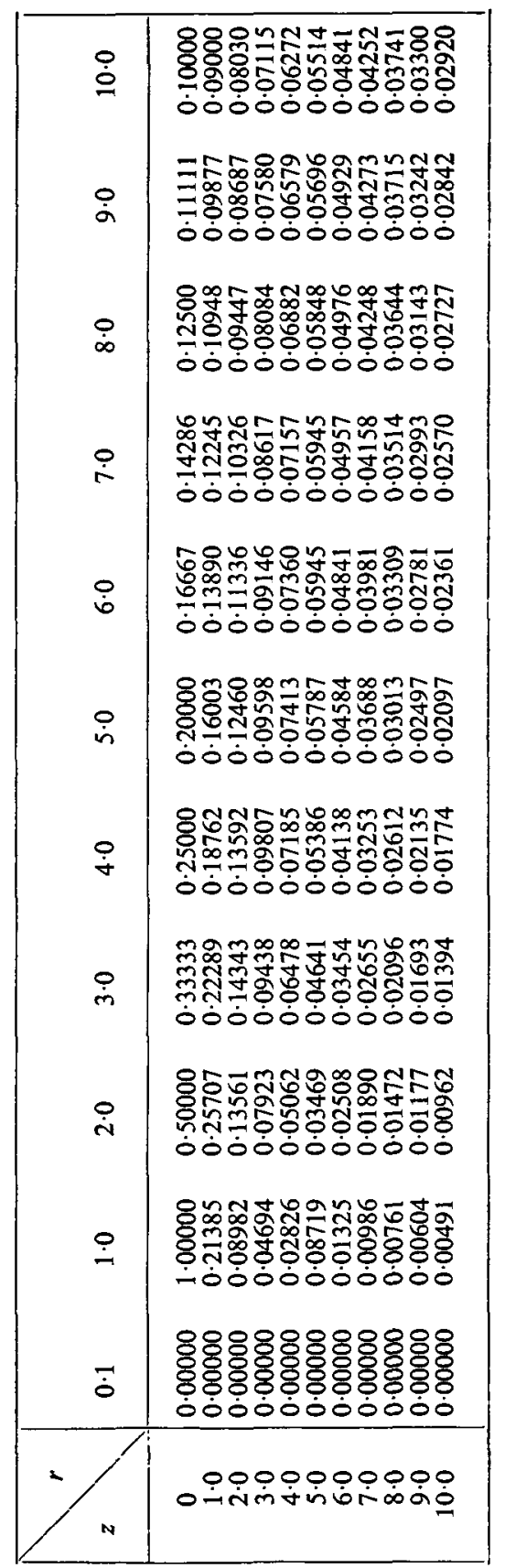




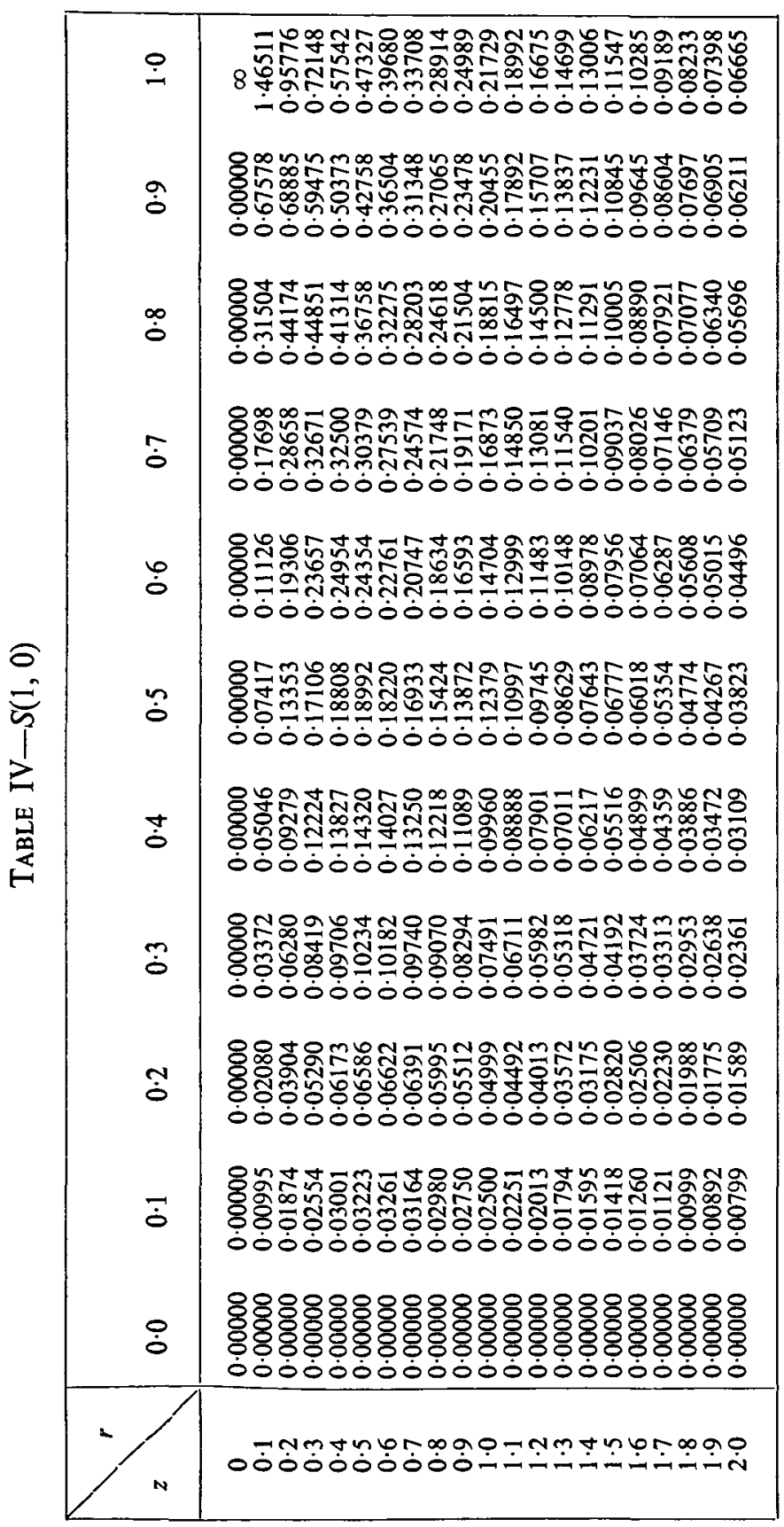


SOME INTEGRALS INVOLVING BESSEL FUNCTIONS

101

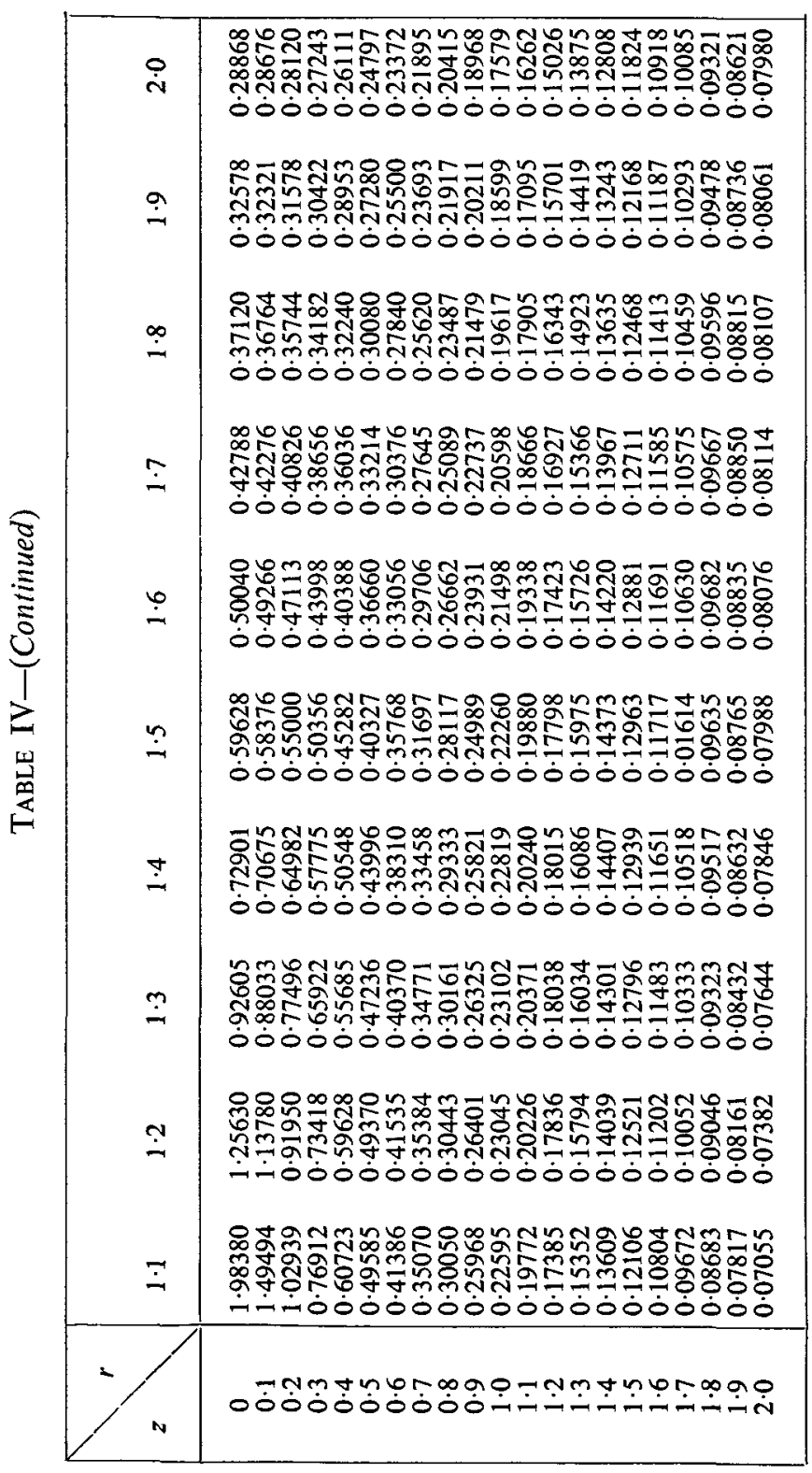

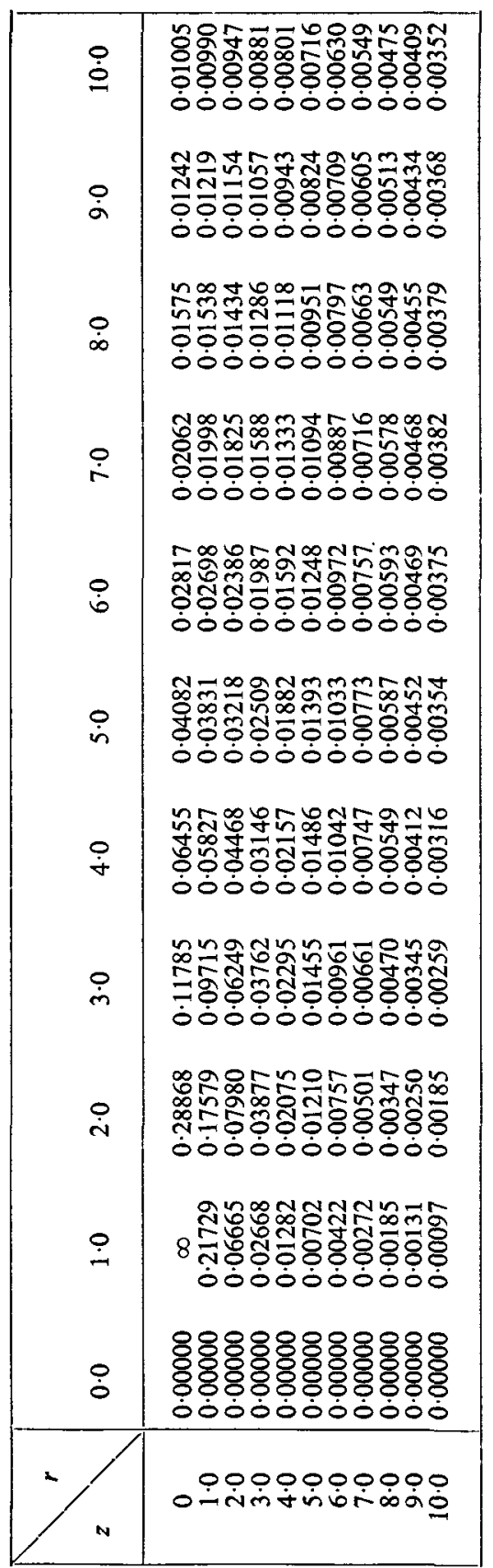




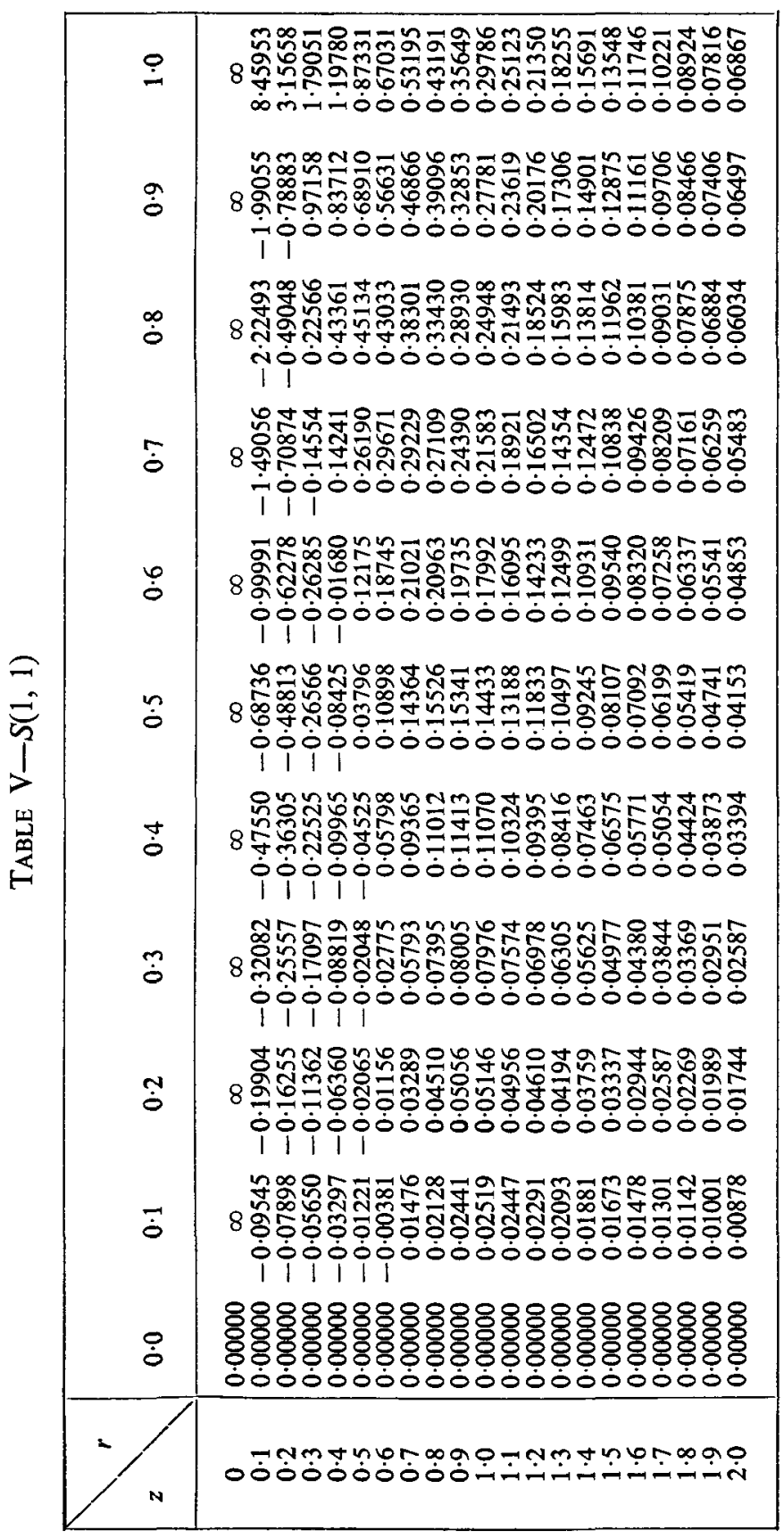




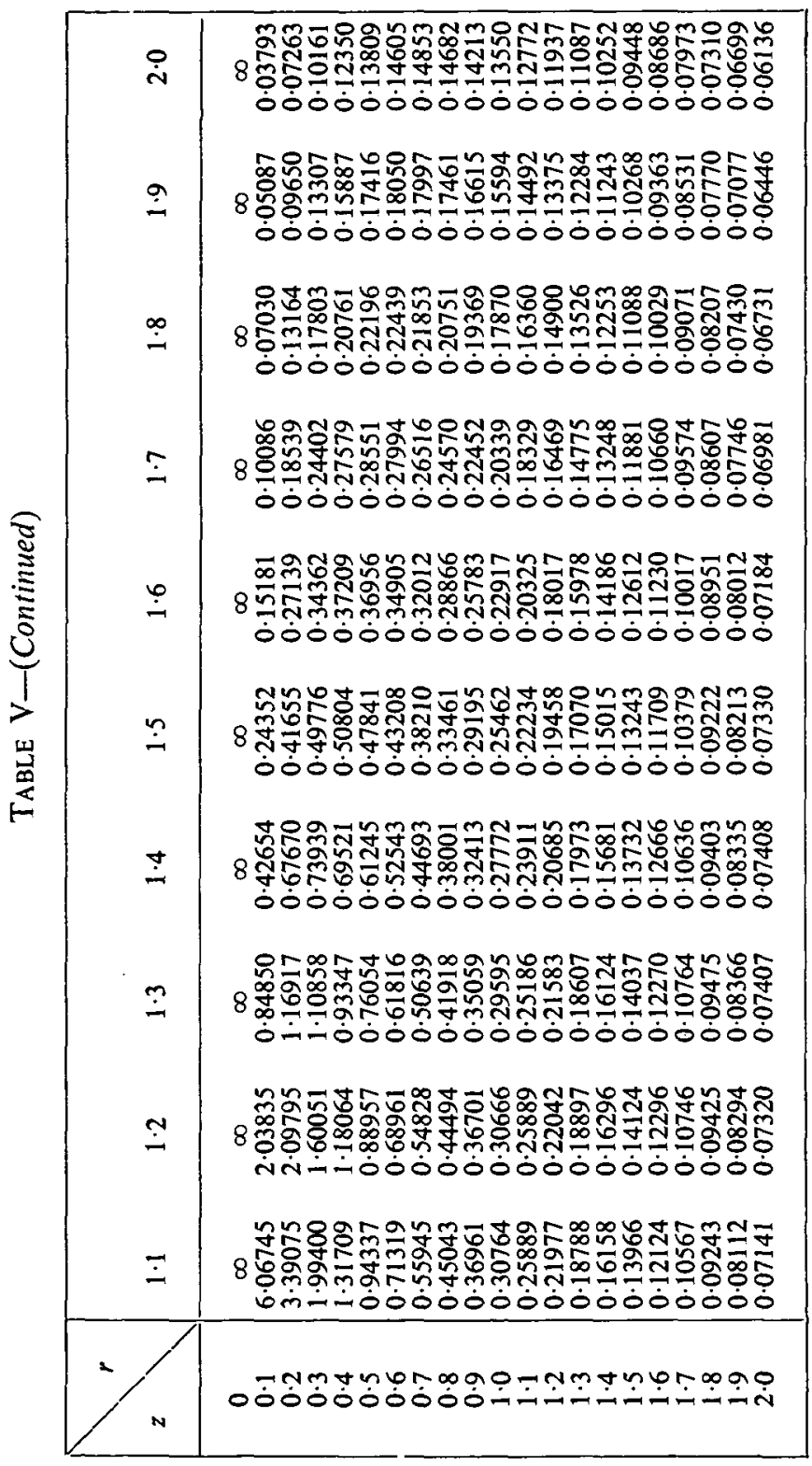

\begin{tabular}{|c|c|}
\hline$\stackrel{\circ}{0}$ & 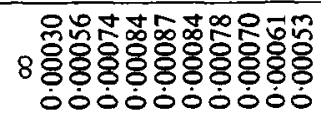 \\
\hline$\stackrel{\circ}{0}$ & 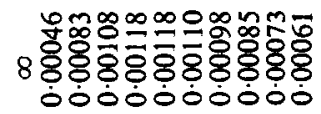 \\
\hline$\stackrel{\infty}{\infty}$ & 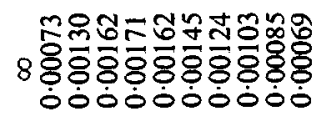 \\
\hline$\stackrel{0}{r}$ & 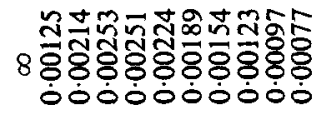 \\
\hline$\stackrel{\phi}{0}$ & 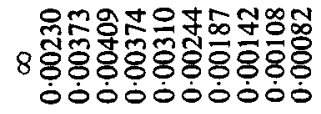 \\
\hline$\stackrel{\dot{\varphi}}{\dot{n}}$ & 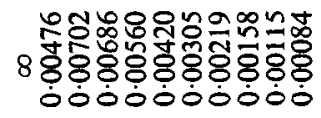 \\
\hline$\stackrel{\varphi}{\dot{q}}$ & 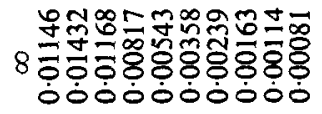 \\
\hline$\stackrel{9}{m}$ & 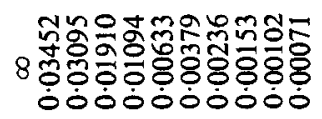 \\
\hline$\stackrel{\circ}{\dot{v}}$ & 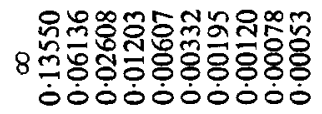 \\
\hline 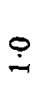 & 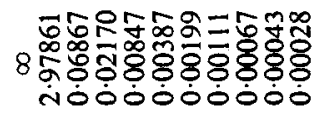 \\
\hline$\dot{0}$ & 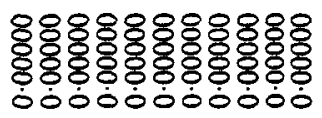 \\
\hline & ○ \\
\hline
\end{tabular}




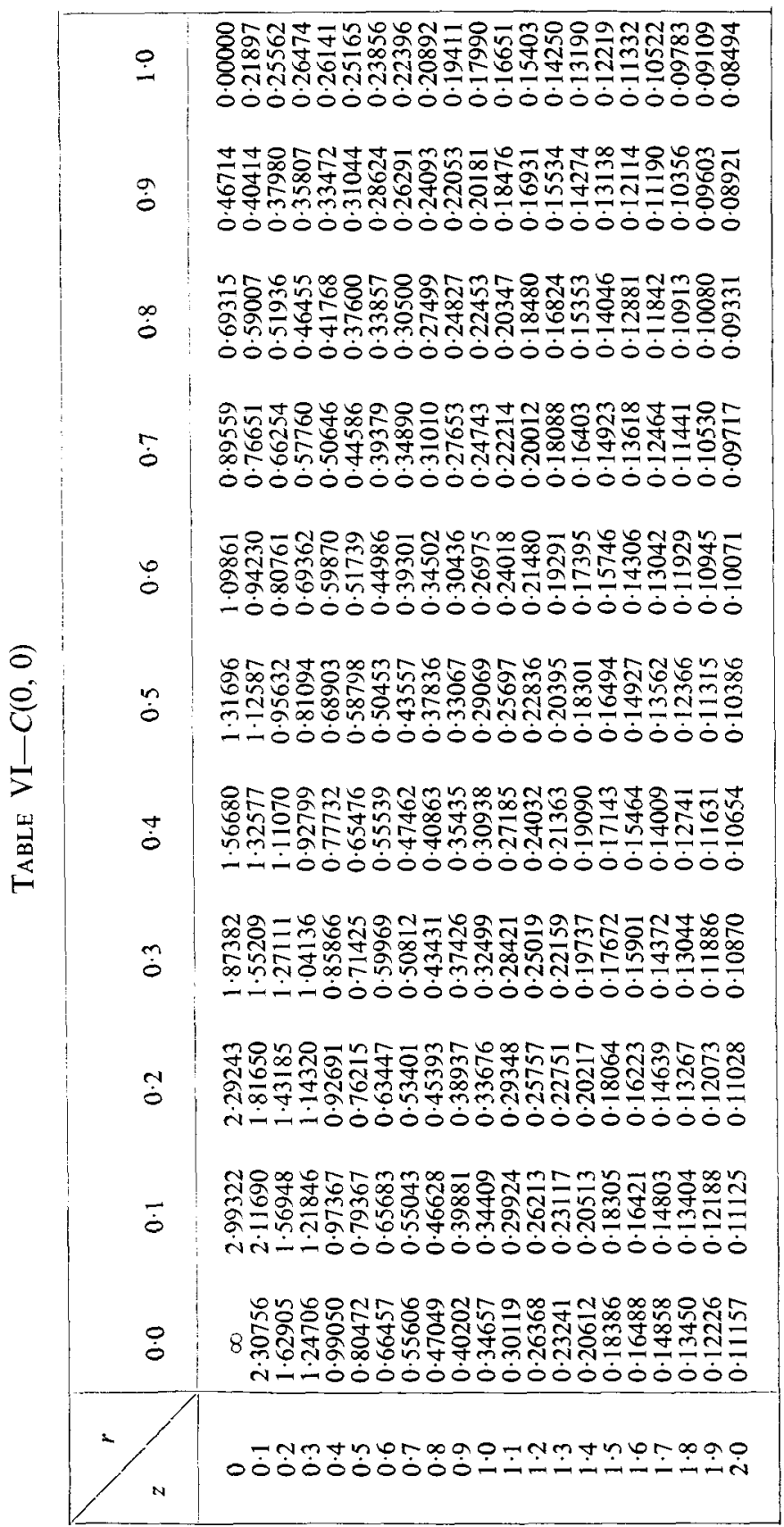




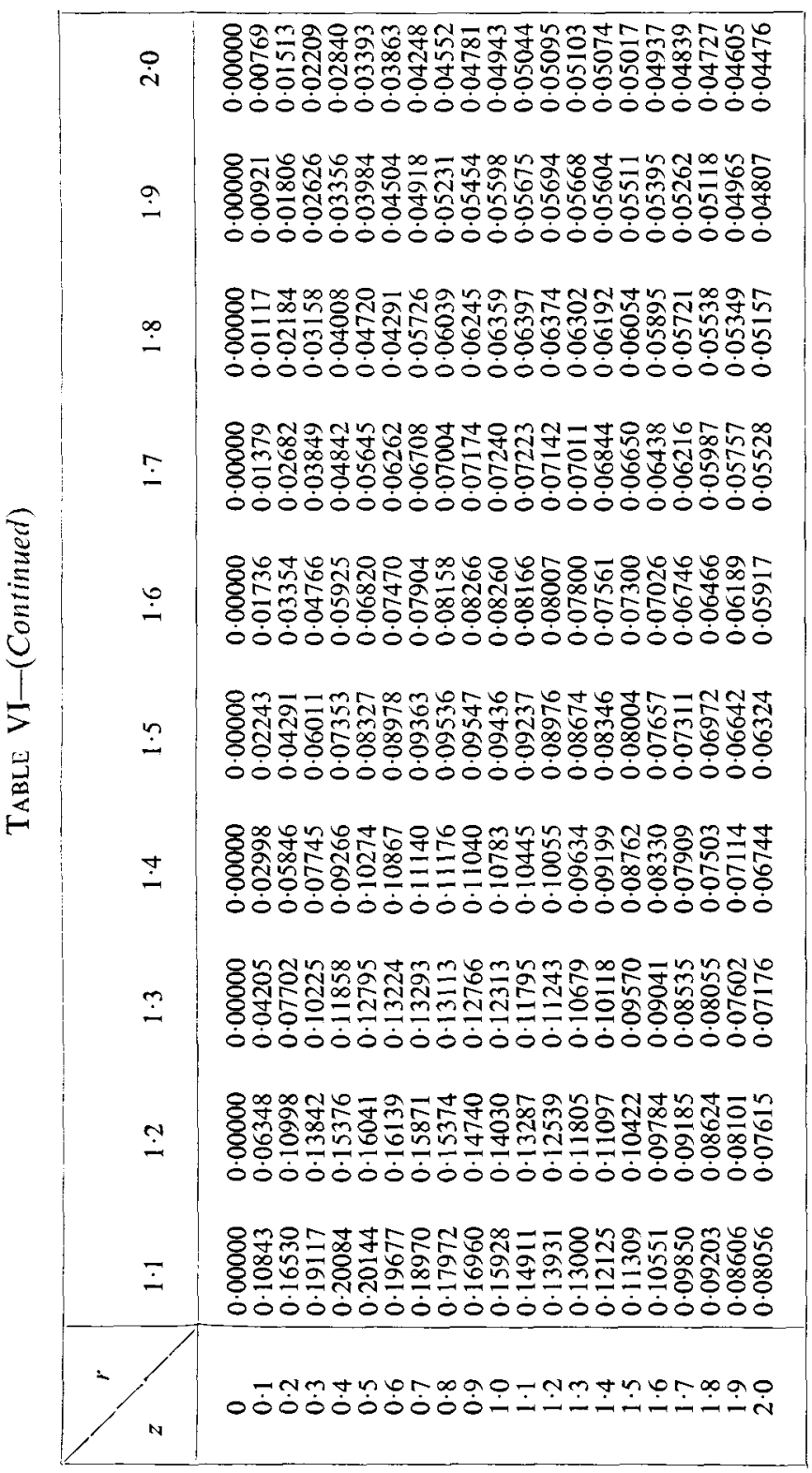

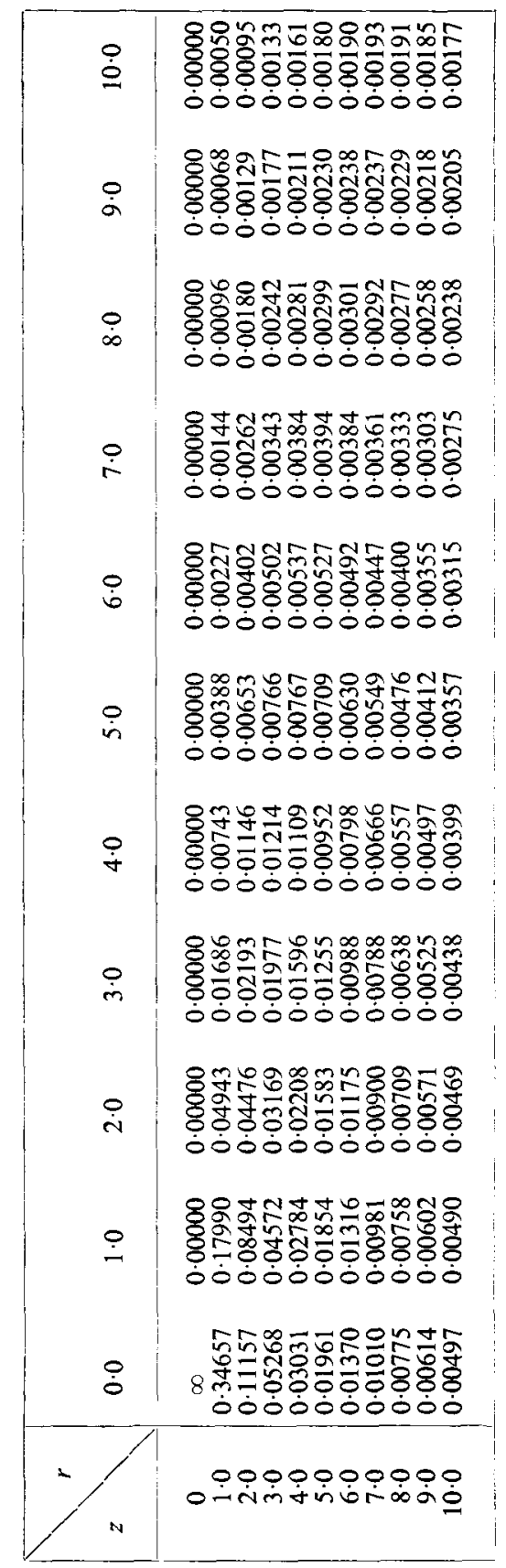




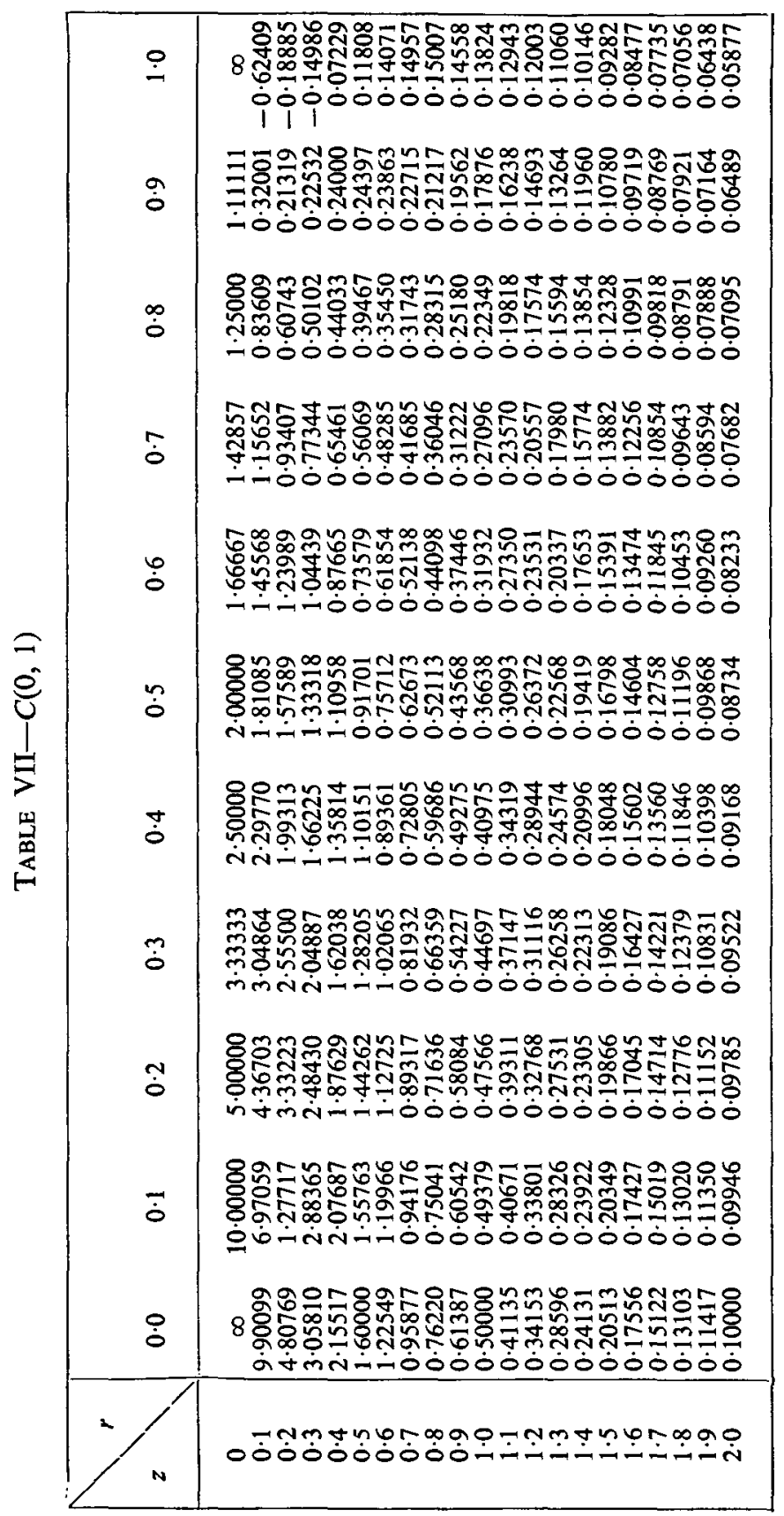




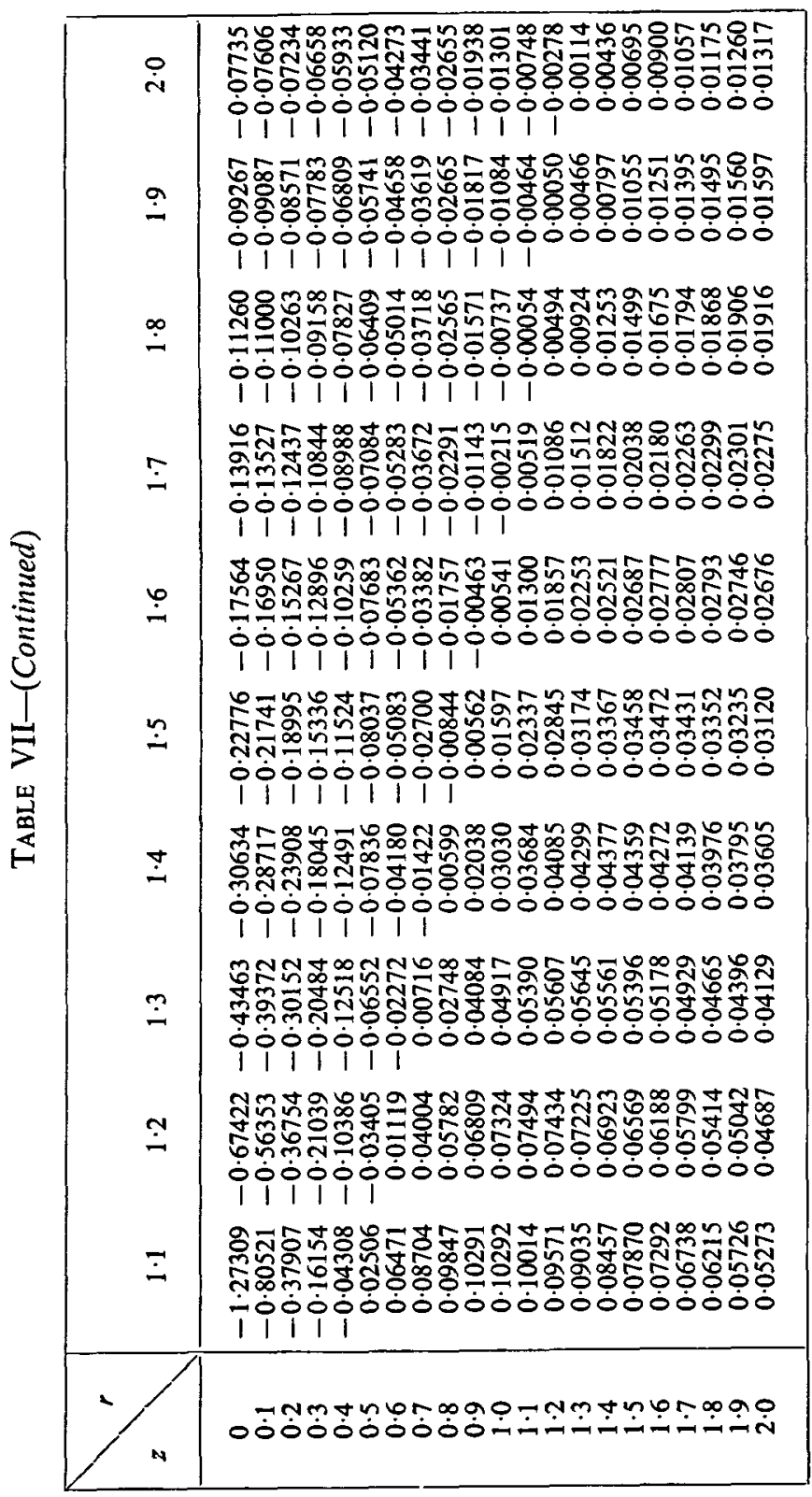

\begin{tabular}{|c|c|}
\hline$\stackrel{\stackrel{\rho}{0}}{2}$ & 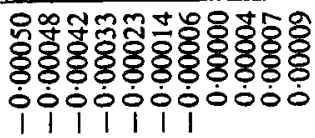 \\
\hline$\stackrel{\circ}{\circ}$ & 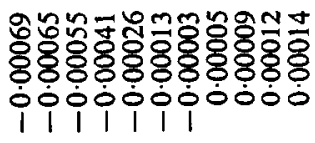 \\
\hline$\stackrel{\varphi}{\infty}$ & 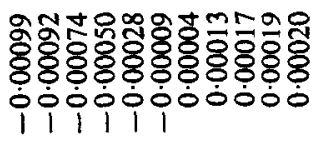 \\
\hline$\stackrel{\varphi}{i}$ & 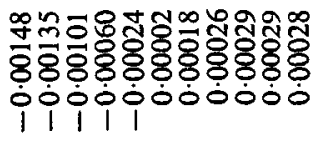 \\
\hline$\stackrel{\varphi}{\dot{0}}$ & 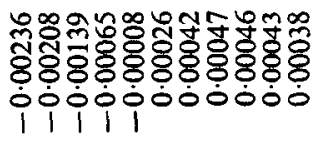 \\
\hline$\dot{\varphi}$ & 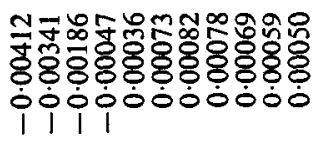 \\
\hline$\stackrel{\varphi}{\dot{q}}$ & 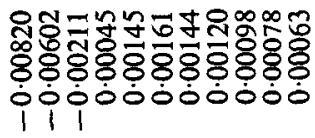 \\
\hline 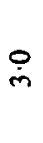 & 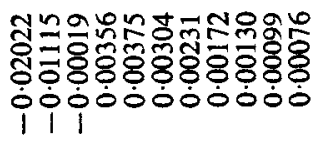 \\
\hline$\stackrel{\circ}{\circ}$ & 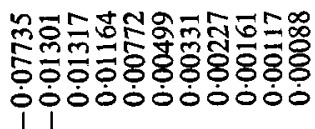 \\
\hline$\stackrel{0}{-1}$ & 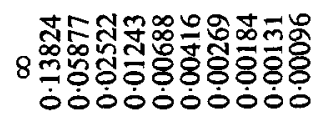 \\
\hline$\dot{0}$ & 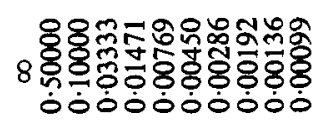 \\
\hline & 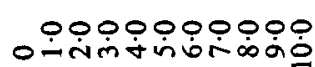 \\
\hline
\end{tabular}




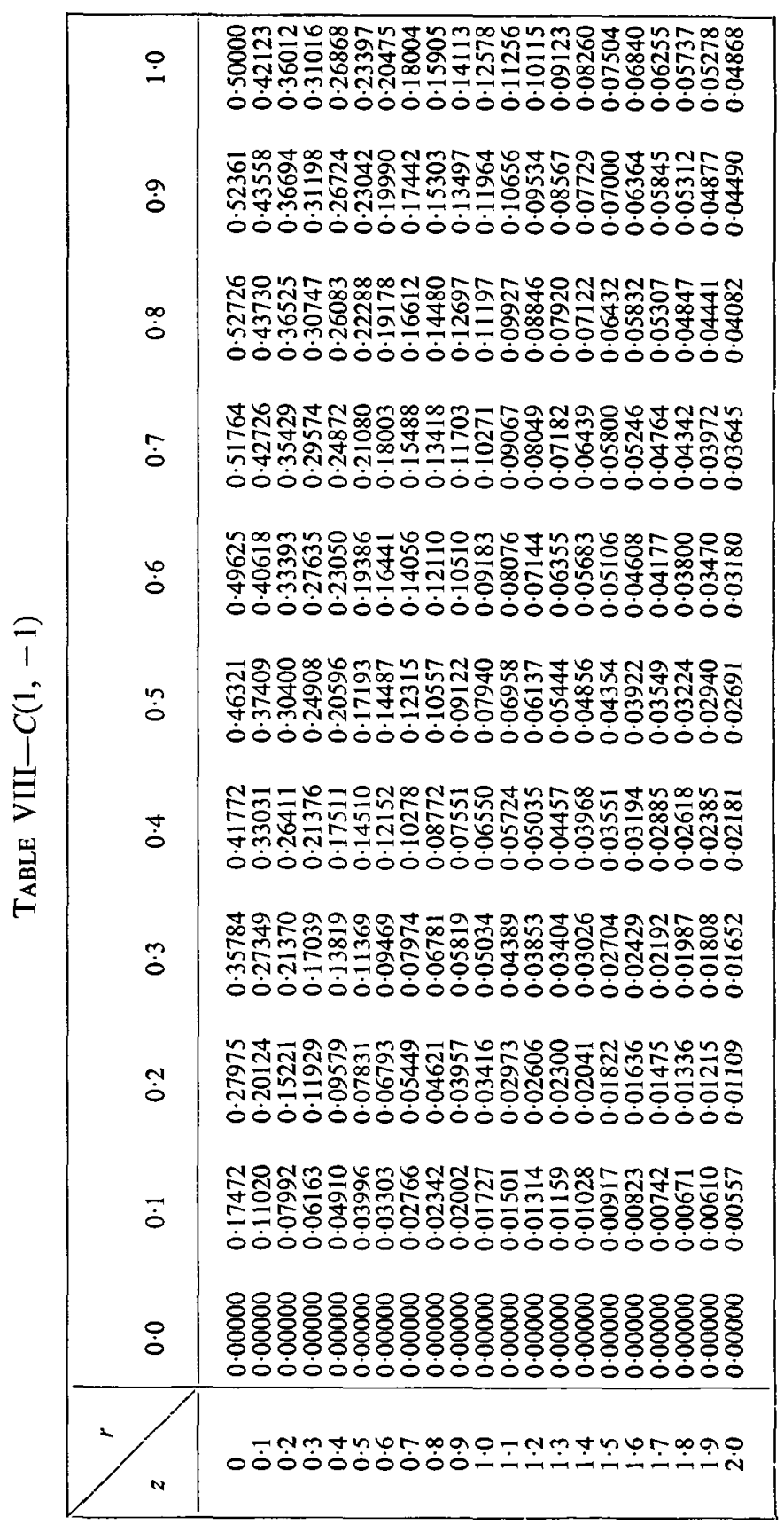




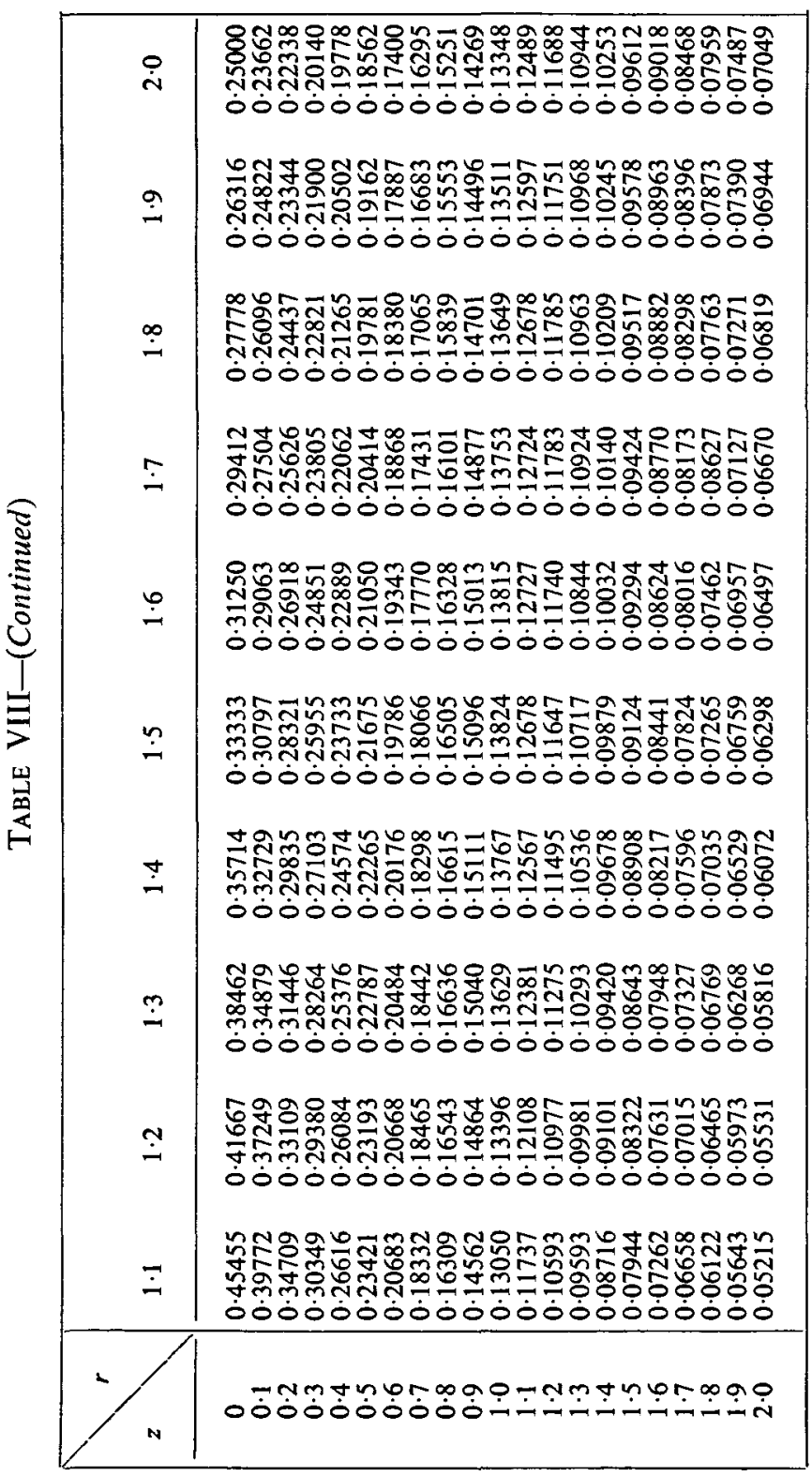

\begin{tabular}{|c|c|}
\hline 잉 & 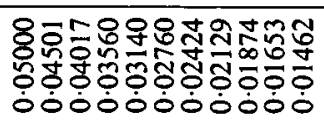 \\
\hline 여 & 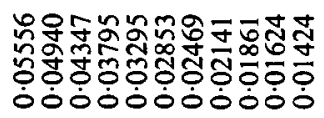 \\
\hline$\stackrel{\leftrightarrow}{\infty}$ & 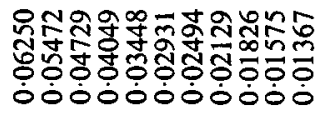 \\
\hline$\stackrel{\leftrightarrow}{1}$ & 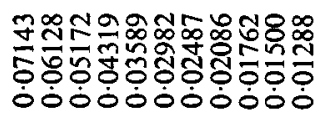 \\
\hline ம) & 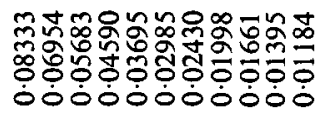 \\
\hline in & 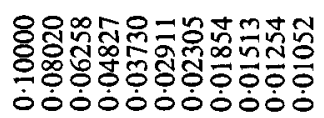 \\
\hline$\stackrel{\dot{q}}{\dot{q}}$ & 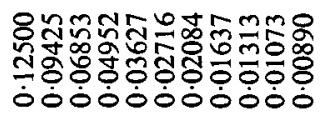 \\
\hline$\stackrel{P}{\dot{m}}$ & 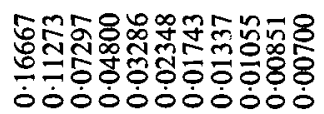 \\
\hline O & 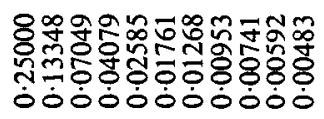 \\
\hline & 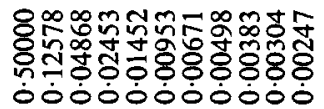 \\
\hline$\stackrel{\varphi}{\dot{0}}$ & 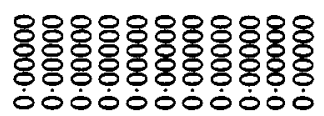 \\
\hline & 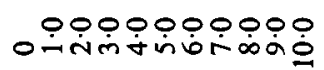 \\
\hline
\end{tabular}




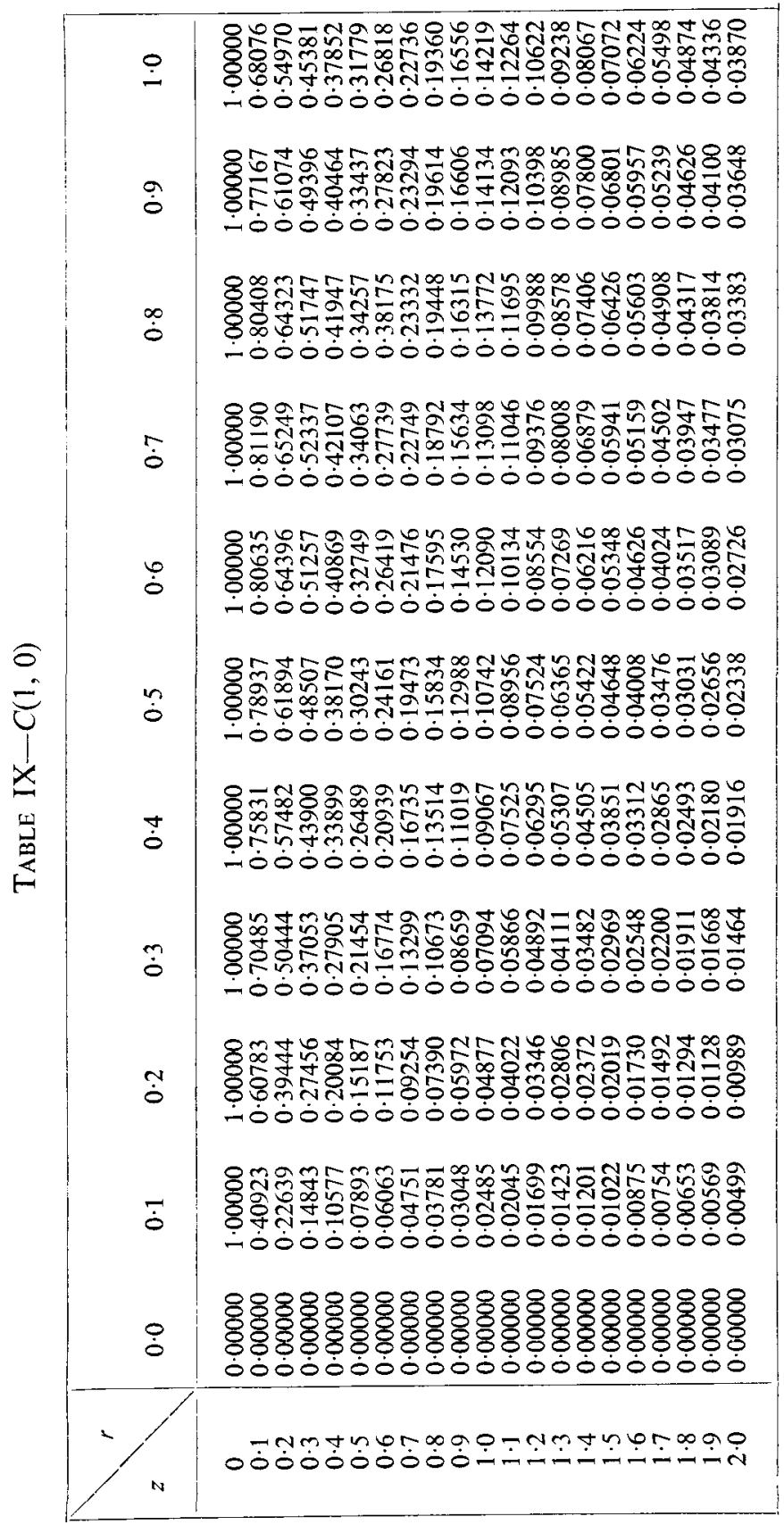




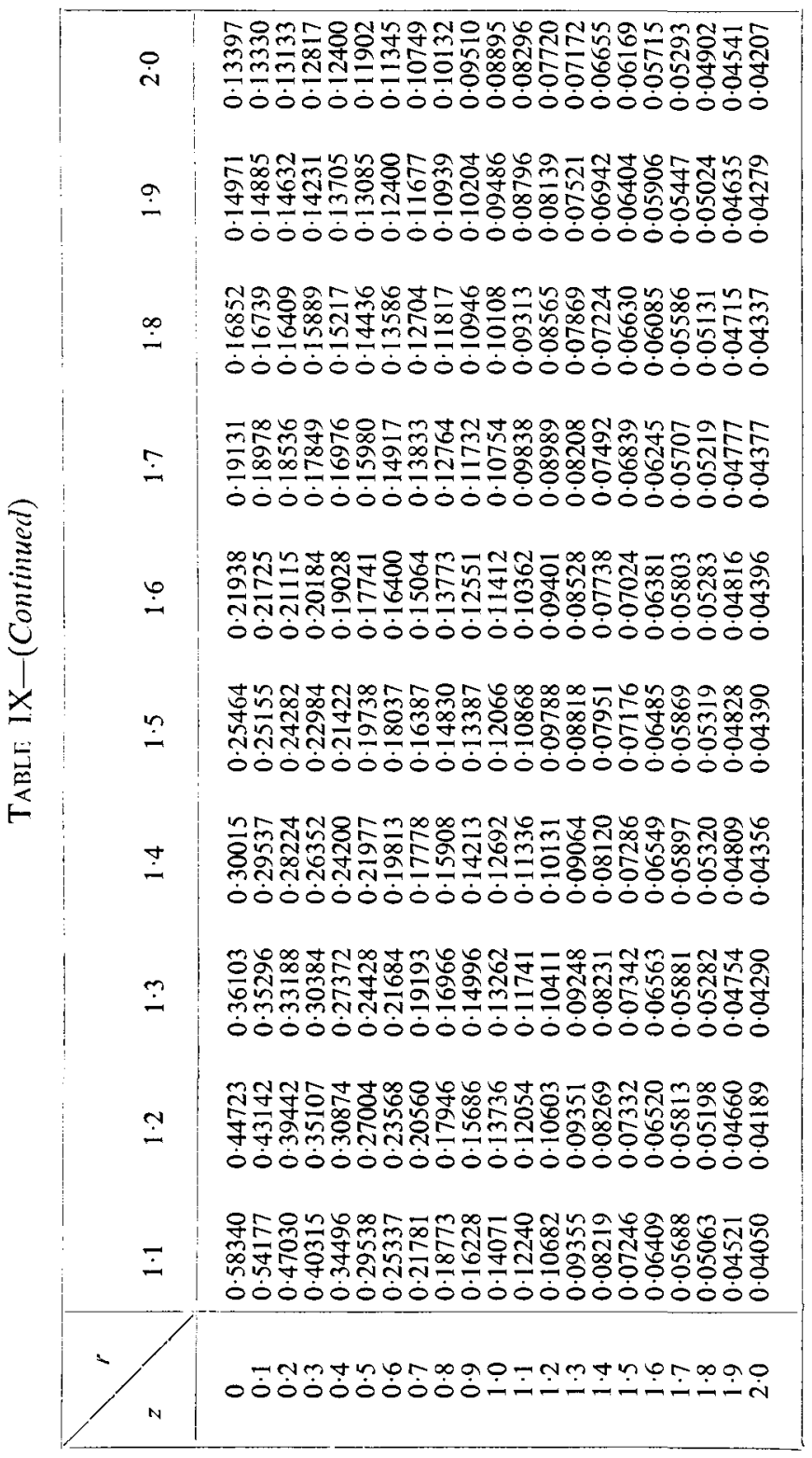

\begin{tabular}{|c|c|}
\hline$\stackrel{\dot{\rho}}{\circ}$ & 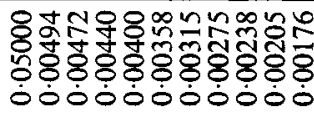 \\
\hline$\stackrel{0}{\dot{\alpha}}$ & 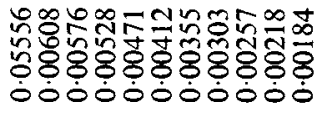 \\
\hline$\underset{\infty}{\infty}$ & 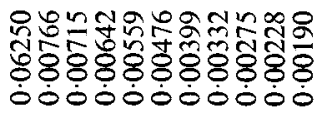 \\
\hline 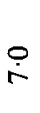 & 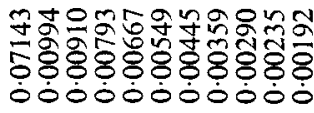 \\
\hline$\stackrel{0}{\dot{0}}$ & 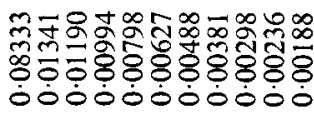 \\
\hline$\stackrel{\varphi}{\dot{n}}$ & 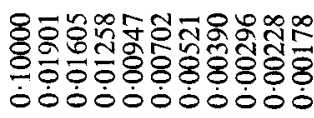 \\
\hline$\stackrel{\stackrel{0}{\dot{v}}}{ }$ & 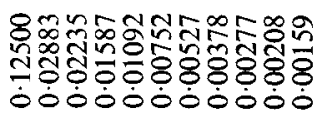 \\
\hline$\dot{m}$ & 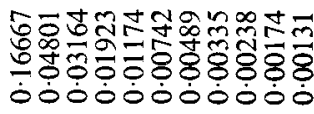 \\
\hline$\stackrel{\dot{n}}{\dot{n}}$ & 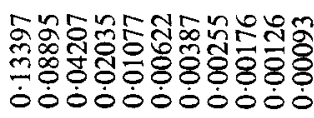 \\
\hline 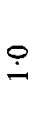 & 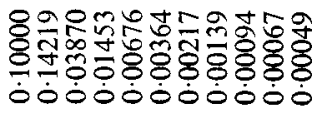 \\
\hline$\dot{0}$ & 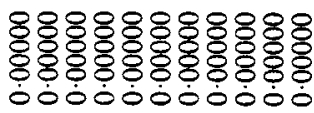 \\
\hline & \\
\hline
\end{tabular}




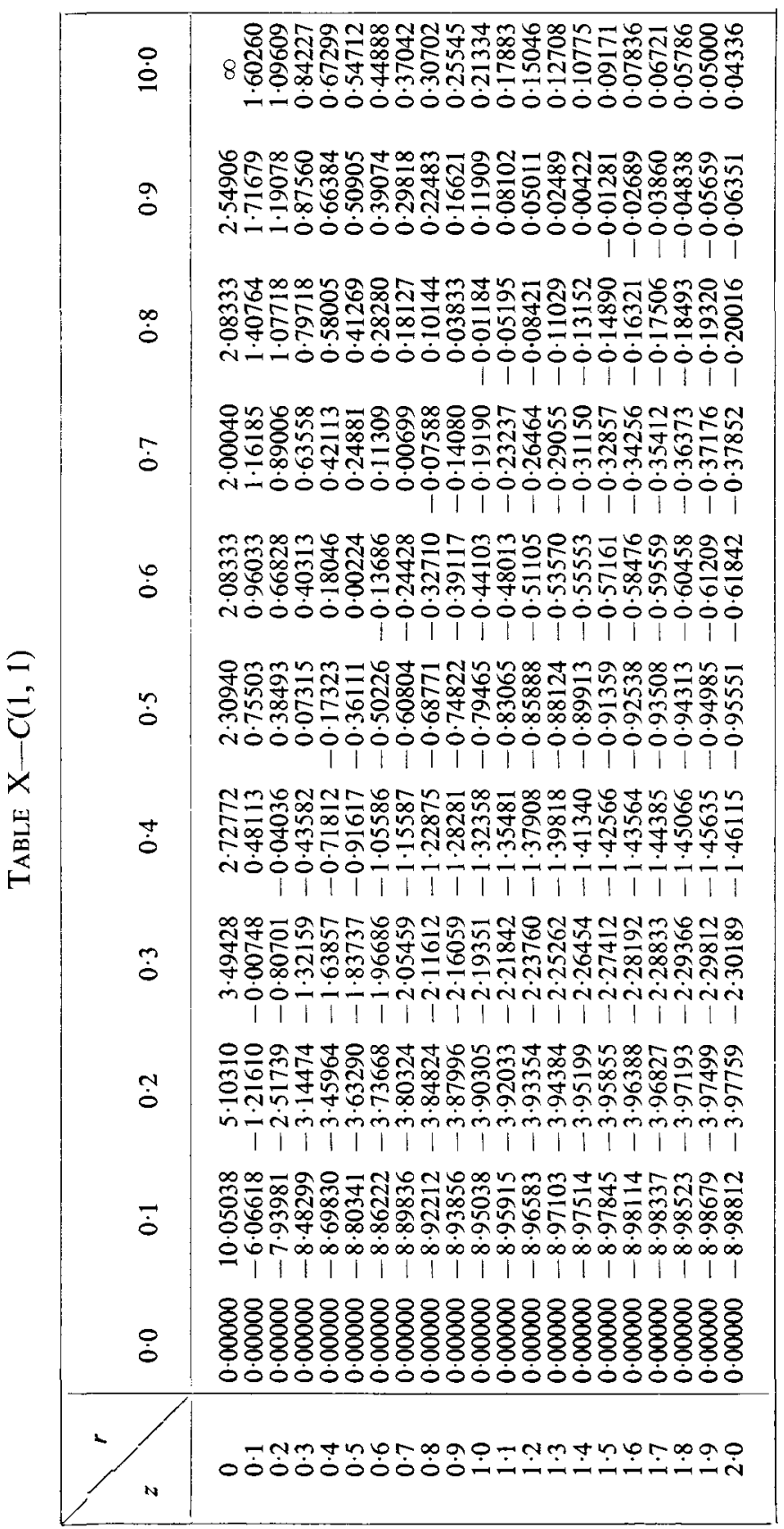




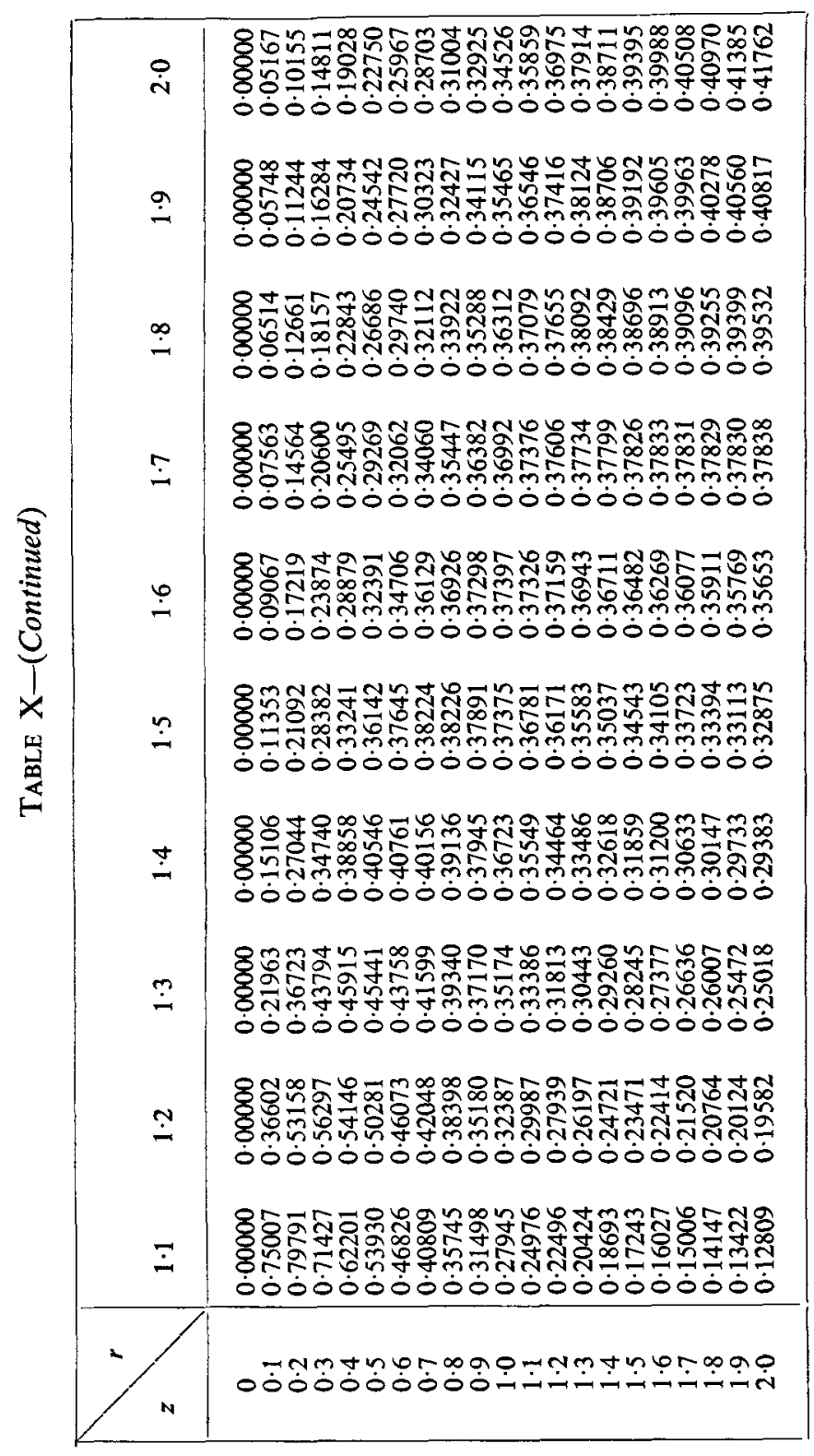

\begin{tabular}{|c|c|}
\hline 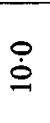 & 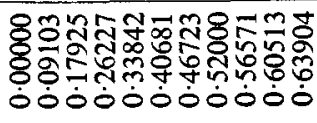 \\
\hline : & 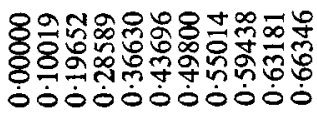 \\
\hline$\stackrel{\varphi}{\infty}$ & 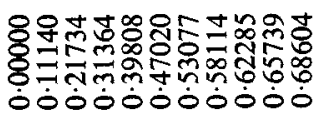 \\
\hline 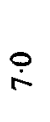 & 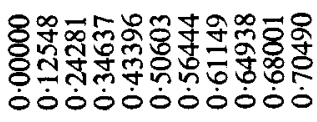 \\
\hline$\dot{0}$ & 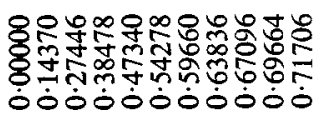 \\
\hline$\stackrel{\varphi}{\dot{n}}$ & 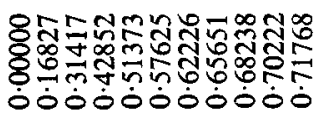 \\
\hline$\stackrel{\rho}{\dot{j}}$ & 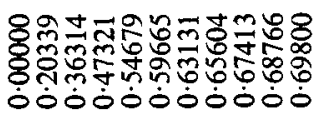 \\
\hline : & 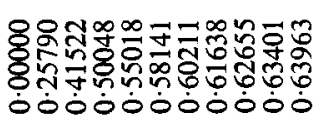 \\
\hline$\stackrel{\dot{\sim}}{ }$ & 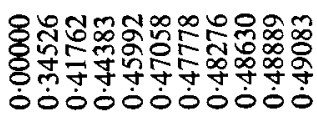 \\
\hline$\stackrel{?}{-}$ & 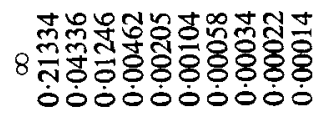 \\
\hline$\overline{0}$ & 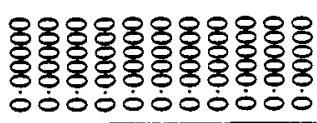 \\
\hline & 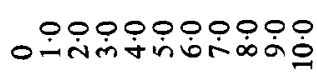 \\
\hline
\end{tabular}

E.M.S.-H 